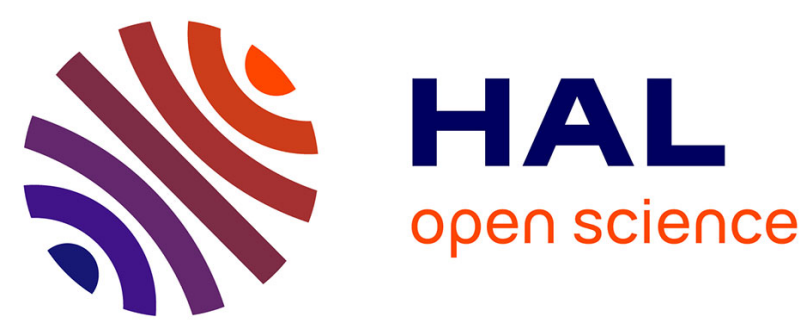

\title{
Étude d'une nouvelle méthode permettant d'orienter, par pompage optique, des niveaux atomiques excités. Application à la mesure de la structure hyperfine de niveaux $1 \mathrm{D}$ de $3 \mathrm{He}$
}

Milica Pavlović, Franck Laloë

\section{To cite this version:}

Milica Pavlović, Franck Laloë. Étude d'une nouvelle méthode permettant d'orienter, par pompage optique, des niveaux atomiques excités. Application à la mesure de la structure hyperfine de niveaux 1D de 3He. Journal de Physique, 1970, 31 (2-3), pp.173-194. 10.1051/jphys:01970003102-3017300 . jpa-00206891

\section{HAL Id: jpa-00206891 https://hal.science/jpa-00206891}

Submitted on 1 Jan 1970

HAL is a multi-disciplinary open access archive for the deposit and dissemination of scientific research documents, whether they are published or not. The documents may come from teaching and research institutions in France or abroad, or from public or private research centers.
L'archive ouverte pluridisciplinaire HAL, est destinée au dépôt et à la diffusion de documents scientifiques de niveau recherche, publiés ou non, émanant des établissements d'enseignement et de recherche français ou étrangers, des laboratoires publics ou privés. 


\title{
ÉTUDE D'UNE NOUVELLE MÉTHODE PERMETTANT D'ORIENTER, PAR POMPAGE OPTIQUE, DES NIVEAUX ATOMIQUES EXCITÉS. APPLICATION A LA MESURE DE LA STRUCTURE HYPERFINE DE NIVEAUX ${ }^{1} D$ DE ${ }^{3} \mathrm{HE}$
}

\author{
Milica PAVLOVIĆ $\left({ }^{1}\right)$ et Franck LALOË \\ Laboratoire de Spectroscopie Hertzienne de l'E. N. S., associé au C. N. R. S., \\ Faculté des Sciences de Paris, France
}

(Reçu le 27 octobre 1969)

\begin{abstract}
Résumé. - On expose dans cet article une nouvelle méthode permettant d'orienter des états atomiques excités ; cette méthode fait intervenir les trois processus suivants :

- on crée par pompage optique une orientation nucléaire dans l'état fondamental des atomes d'une vapeur ;

- une décharge H. F. porte les atomes dans divers états excités, sans détruire leur orientation nucléaire.

- l'orientation nucléaire ainsi obtenue dans chaque niveau excité peut être transformée en orientation électronique, par l'intermédiaire du couplage hyperfin aI.J ; la lumière émise par la décharge est alors polarisée.

L'étude théorique de l'orientation électronique obtenue par cette méthode a été effectuée, de façon à mettre en évidence l'influence des diverses caractéristiques du niveau excité, ainsi que celle du champ magnétique.

Cette méthode a été utilisée pour orienter divers niveaux excités de ${ }^{3} \mathrm{He}$. Une étude détaillée a été effectuée pour les niveaux $3{ }^{1} D, 4^{1} D$ et $5^{1} D$, notamment dans le cas où un champ magnétique statique introduit un découplage hyperfin partiel; ces expériences ont conduit à une mesure de la structure hyperfine de ces trois niveaux.
\end{abstract}

Abstract. - A new mathod for orienting excited states is reported ; the three following processes are involved:

- a nuclear orientation in the ground state of the atoms is achieved by optical pumping;

- a R.F. discharge in the vapour populates the various excited levels of the atoms, without destroying the orientation of the nuclei ;

- in each excited state, this nuclear orientation can be transformed by the hyperfine coupling aI.J in an electronic orientation, which can be easily detected by the polarisation of the emitted light.

Theoritical study of the electronic orientation obtained by this method is presented as a function of the parameters of the excited state and of the static magnetic field.

This method has been used to orient various excited levels of ${ }^{3} \mathrm{He}$. A detailed study of the effect of the hyperfine decoupling in the $3^{1} D, 4^{1} D$ and $5^{1} D$ levels has shown a good agreement between experimental results and theory, and yields a measurement of the hyperfine structure of these levels.

Introduction. - Les expériences de pompage optique sont souvent réalisées avec des atomes dont le spin nucléaire I n'est pas nul [1] ; lorsque l'état fondamental des atomes est diamagnétique (moment angulaire électronique $J=0$ ), elles permettent même d'obtenir une orientation purement nucléaire, comme par exemple dans le cas du mercure [2], du cadmium [3] et du zinc [4]. Le spin $\mathbf{I}$ joue alors un rôle très particulier ; en effet, la méthode du pompage optique consiste

(1) Institut “ Ruder Bošković », Zagreb, Yougoslavie. LE JOURNAL DE PHYSIQUE. - T. 31, No 2-3, FÉVRIER-MARS 1970. à envoyer sur les atomes une onde lumineuse convenablement polarisée qui interagit avec les électrons de l'atome, mais dont l'action sur le spin nucléaire est complètement négligeable. Le spin nucléaire n'est donc pas directement affecté par cette méthode, et on peut même se demander comment il est possible d'obtenir une orientation nucléaire par pompage optique.

Cette question a été étudiée de manière approfondie par Lehmann [5] ; cet auteur a généralisé les travaux théoriques antérieurs relatifs au cycle de pompage optique [6], [7], de façon à mettre en évidence d'une 
façon plus détaillée le rôle joué par le spin nucléaire $\mathbf{I}$; il a pu ainsi montrer que, dans une expérience de pompage optique, une orientation nucléaire ne peut être obtenue que par l'intermédiaire du couplage hyperfin $a \mathbf{I . J}$; en effet ce couplage fait passer l'orientation du moment angulaire électronique $\mathbf{J}$, obtenue par interaction avec le faisceau lumineux polarisé, au moment nucléaire I. Pour rappeler de manière plus précise les résultats de Lehmann, il est utile de considérer le « cycle de pompage » suivi par les atomes ; on sait que, dans les conditions habituelles, ce cycle comporte quatre étapes successives et indépendantes :

- Absorption d'un photon de résonance optique polarisé, au cours de laquelle l'atome passe de l'état fondamental à l'état excité.

- Evolution propre dans l'état excité.

- Emission spontanée qui fait retomber l'atome dans l'état fondamental.

- Evolution propre dans l'état fondamental.

On peut associer à chacune de ces étapes un temps $\Delta t$, qui constitue un ordre de grandeur de la durée des phénomènes transitoires correspondants. Par exemple, au processus d'absorption est associé un temps $\Delta t \simeq 1 / \Delta(\Delta$ est la largeur spectrale du faisceau lumineux excitateur, exprimée en unités de pulsation): $1 / \Delta$ représente le temps de passage d'un paquet d'ondes du faisceau lumineux en un point fixe de l'espace. De même, à l'évolution propre dans l'état excité est associé un temps $\Delta t \simeq \tau(\tau$ durée de vie de l'état excité correspondant).

Pour que le spin nucléaire soit affecté au cours de l'une de ces étapes il suffit que, pendant le temps $\Delta t$ correspondant, le couplage hyperfin dans l'un des niveaux atomiques concernés soit assez grand pour agir sur I ; par exemple, l'évolution propre dans l'état excité peut orienter $\mathbf{I}$ lorsque la condition $a \tau \gtrsim 1$ est réalisée ( $a$ est la constante de couplage hyperfin dans le niveau considéré, exprimée en unités de pulsation), et bien sûr lorsque $\mathbf{J}$ a préalablement été orienté. L'orientation de $\mathbf{I}$ ainsi obtenue n'est pas affectée par le processus d'émission spontanée, au cours duquel la polarisation nucléaire peut donc être transférée à l'état fondamental : c'est une des méthodes classiques qui permet d'orienter un ensemble de spins nucléaires par pompage optique.

La nouvelle méthode que nous étudions dans cet article présente une certaine analogie avec celle qui vient d'être rappelée. Considérons une vapeur formée d'atomes dont le spin nucléaire a été orienté dans l'état fondamental; on peut penser à utiliser le fait que le spin I est peu couplé au milieu extérieur pour transférer son orientation dans divers niveaux atomiques. Supposons en effet que l'atome subisse un processus d'excitation très court qui n'agisse directement que sur ses variables électroniques; ce processus n'affectera pas, comme nous venons de le voir, l'orientation du spin I ; l'atome conservera donc en arrivant dans l'état excité l'orientation nucléaire qu'il avait dans l'état fondamental. Si la durée de vie du niveau excité est suffisamment longue pour permettre au couplage hyperfin de s'établir, cette orientation nucléaire pourra être partiellement transformée en orientation électronique. Le mécanisme qui vient d'être décrit fournit donc une nouvelle méthode d'orientation du moment électronique $\mathbf{J}$; cette méthode comprend quatre étapes, qui correspondent aux étapes décrites plus haut, mais prises dans l'ordre inverse :

- On oriente par pompage optique le spin nucléaire dans l'état fondamental.

- Un processus de collision très rapide (que nous préciserons plus bas de manière plus détaillée) permet d'exciter l'atome, sans toutefois détruire son orientation nucléaire. Remarquons qu'il est possible de supposer que l'excitation des atomes est isotrope, et qu'elle ne crée donc ni orientation ni alignement électronique.

- Au cours de l'évolution propre dans l'état excité, l'orientation de $\mathbf{I}$ se transmet en partie à $\mathbf{J}$ par l'intermédiaire du couplage hyperfin $a \mathbf{I}$.J, pourvu toutefois que la durée de vie $\tau$ soit suffisamment longue (condition $a \tau \gtrsim 1$ ).

- L'atome perd son excitation par émission spontanée. L'orientation acquise par $\mathbf{J}$ apparaît alors sous la forme d'une polarisation de la lumière de fluorescence émise $\left({ }^{2}\right)$.

Tout le raisonnement précédent repose sur le fait qu'une orientation nucléaire n'est pas affectée lors d'une collision agissant uniquement sur les électrons et durant un temps extrêmement court $\left(^{3}\right)$. Cette propriété importante en physique atomique a effectivement été vérifiée dans de nombreux autres cas analogues; citons par exemple l'étude des collisions d'atomes ${ }^{199} \mathrm{Hg}$ ou ${ }^{201} \mathrm{Hg}$ dans l'état $6{ }^{3} P_{1}$ contre des atomes de gaz rares [8] ou contre des atomes de $\mathbf{H g}$ dans l'état fondamental [9], qui montre que I n'est pas affecté lors du processus "instantané 》 de collision (alors que l'atmosphère électronique est modifiée) ; de même, l'étude des collisions d'échange entre deux alcalins [10] montre que les deux spins électroniques s'échangent sans que les spins nucléaires soient affectés.

(2) Notons que, même si l'orientation de l'état excité était purement nucléaire, il existerait déjà une polarisation lumineuse de chacune des composantes hyperfines de la raie étudiée ; toutefois les divers effets de la polarisation nucléaire s'annuleraient entre eux sur l'ensemble des composantes, de sorte que la lumière de fluorescence globale ne serait pas polarisée (cf. § I, $\mathrm{B}, 1)$. La séparation des composantes hyperfines étant expérimentalement difficile à réaliser, nous supposons dans tout cet article que c'est cette lumière globale qui est observée ; dans ces conditions, l'orientation de $\mathbf{J}$ est indispensable pour voir apparaître une polarisation lumineuse.

(3) Les structures hyperfines que l'on rencontre en physique atomique peuvent êtretrès faibles (nulles ou inférieures à $1 \mathrm{MHz}$, comme par exemple dans certains niveaux de ${ }^{3} \mathrm{He}$ ) et peuvent atteindre quelques dizaines de milliers de Mégahertz (cas par exemple du mercure). Dire que la collision dure un temps très court revient donc à dire que le temps de collision $\tau_{\mathrm{c}}$ est inférieur à $10^{-11}$ seconde. 
Nous avons vu plus haut que l'excitation des atomes peut avoir un caractère isotrope ; il n'est donc pas nécessaire de recourir aux techniques de bombardement électronique [11] qui, grâce à la directivité d'un faisceau d'électrons, permettent de produire directement un alignement électronique dans un niveau atomique excité. Expérimentalement, il est beaucoup plus commode d'entretenir une décharge dans la vapeur atomique, ce qui permet d'atteindre aisément un grand nombre de niveaux. Une décharge dans un gaz sous une pression de l'ordre du torr possède un caractère isotrope : en effet les électrons subissent à cette pression de très nombreuses collisions qui dévient constamment leurs trajectoires; il s'ensuit que les collisions qui excitent les atomes ont lieu avec une probabilité pratiquement égale dans toutes les directions. (Rappelons que les pressions utilisées dans des expériences de bombardement électronique sont beaucoup plus faibles ; par exemple, dans le cas de l'Hélium, ces pressions sont de l'ordre de $10^{-2}$ à $10^{-4}$ torr [12].) L'excitation des atomes par une décharge semble donc être bien adaptée à l'obtention d'une orientation du moment angulaire électronique $\mathbf{J}$ par la nouvelle méthode décrite dans cet article ; c'est donc le procédé d'excitation que nous avons utilisé.

L'utilisation d'une décharge conduit tout naturellement à réaliser les expériences dans le cas de ${ }^{3} \mathrm{He}$ (spin nucléaire $I=\frac{1}{2}$ ) : on sait [13] que l'orientation nucléaire de ${ }^{3} \mathrm{He}$ peut être obtenue par pompage optique du niveau métastable $2{ }^{3} S_{1}$; en effet, il se produit dans la vapeur des collisions dites « d'échange de métastabilité » qui transfèrent l'orientation du niveau métastable à l'état fondamental; pour porter une certaine proportion des atomes dans l'état métastable $2{ }^{3} S_{1}$, on entretient une décharge faible dans l'hélium. On peut donc, dans le cas de ${ }^{3} \mathrm{He}$, vérifier aisément que le procédé d'orientation des états atomiques excités que nous avons décrit existe réellement : il suffit de voir si, lorsque l'on oriente le spin nucléaire dans l'état fondamental, les différentes raies spectrales de la lumière émise par la décharge sont polarisées.

Cette expérience a été réalisée et a donné un résultat positif. Dans cet article, nous allons exposer les divers résultats expérimentaux obtenus, leur comparaison avec les prévisions théoriques, ainsi que la méthode de mesure de structures hyperfines qui a pu ainsi être mise au point.

La première partie de cet article est consacrée à une étude théorique de l'orientation électronique obtenue dans chaque niveau, en fonction de diverses grandeurs intervenant dans le problème : $a$, constante du couplage hyperfin $a \mathbf{I} . \mathbf{J} ; \tau$, durée de vie du niveau excité, etc... L'influence d'un champ magnétique statique appliqué $\mathbf{B}_{0}$ est étudiée. On s'intéresse notamment au cas où $\mathbf{B}_{0}$ introduit dans l'état excité considéré un découplage hyperfin partiel; on montre alors que cet état acquiert non seulement une orientation électronique $\left\langle J_{z}\right\rangle$, mais encore un alignement électronique $<3 J_{z}^{2}-J(J+1)>$ (pour simplifier on suppose que l'orientation nucléaire dans l'état fondamental est longitudinale, c'est-à-dire parallèle à l'axe $O z$ qui porte $\mathbf{B}_{0}$ ).

Dans la seconde partie, nous décrivons les expériences qui ont permis d'appliquer cette nouvelle méthode d'orientation des états atomiques excités au cas de ${ }^{3} \mathrm{He}$. L'influence de divers paramètres qui interviennent dans l'expérience est discutée. On montre ensuite comment il est possible d'obtenir à partir des résultats expérimentaux une mesure de la structure hyperfine de certains niveaux atomiques excités : on donne les résultats obtenus dans le cas des niveaux $3^{1} \mathrm{D}, 4{ }^{1} \mathrm{D}$ et $5^{1} \mathrm{D}$ de ${ }^{3} \mathrm{He}$.

\section{I. ÉTUDE THÉORIQUE.}

A. Position du problème ; étude qualitative. 1. Introduction. Modèle SIMPle. - Considérons un ensemble d'atomes qui, au moment où ils viennent d'être excités, possèdent une certaine orientation nucléaire. Par contre, nous supposons que, juste après leur excitation, leur atmosphère électronique est isotrope (c'est-à-dire que toutes les grandeurs électroniques du type orientation, alignement, etc... sont nulles). Notons que cette hypothèse n'exclut pas la possibilité, pour une collision donnée, de produire un certain alignement atomique, comme cela se produit dans de nombreux cas [11] : une excitation électronique isotrope sera réalisée pourvu que l'ensemble des atomes subisse des collisions aussi nombreuses dans toutes les directions, de sorte qu'en moyenne l'alignement créé reste nul.

Une fois excités, les atomes vont rester dans le niveau où ils se trouvent pendant un certain temps, de l'ordre de la durée de vie $\tau$. Le couplage hyperfin est alors susceptible de modifier l'orientation nucléaire, comme l'orientation électronique. Considérons par exemple le cas le plus simple, celui où les deux nombres quantiques $I$ et $J$ valent $\frac{1}{2}$. Au moment où l'atome vient d'être excité, I pointe dans une direction fixe $O z$ (voir Fig. 1), alors que $\mathbf{J}$ pointe dans une direction aléatoire, de sorte que l'on a $\langle\mathbf{J}\rangle=0$. Au cours de l'évolution propre dans l'état excité, I et $\mathbf{J}$ se couplent pour former $\mathbf{F}=\mathbf{I}+\mathbf{J}$ et, sous l'effet de l'interaction hyperfine $a \mathbf{I} . \mathbf{J}$, précessent autour de cette résultante commune; cette " précession hyperfine » s'effectue avec la vitesse angulaire $a$. Deux cas sont alors possibles :

- lorsque la condition $a \tau \ll 1$ est réalisée, I et $\mathbf{J}$ n'ont pas le temps, pendant la durée de vie $\tau$, d'effectuer une fraction notable de tour autour de $\mathbf{F}$; les deux orientations, nucléaire et électronique, sont donc 


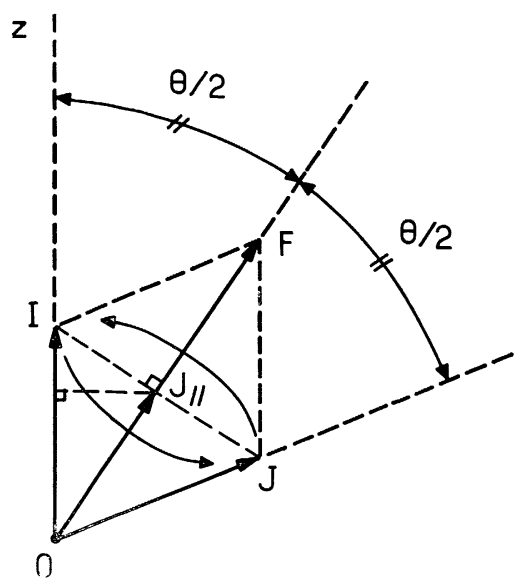

FIG. 1. - Couplage entre les moments cinétiques $\mathbf{I}$ et $\mathbf{J}$, dans le cas ou $I=J=\frac{1}{2}$. La direction $O z$ est fixe, et l'angle $\theta$ entre $\mathbf{J}$ et $O z$ a une valeur aléatoire.

pratiquement inchangées lorsque l'émission spontanée fait retomber l'atome dans un niveau d'énergie plus basse ; l'orientation électronique $\langle\mathbf{J}\rangle$ reste alors nulle en moyenne,

- lorsque $a \tau$ est de l'ordre de 1 (ou plus grand), $\mathbf{I}$ et $\mathbf{J}$ ont le temps d'évoluer avant que l'atome ne quitte le niveau considéré. Etudions par exemple le cas limite où la condition $a \tau \gg 1$ est réalisée ; I et $\mathbf{J}$ effectuent alors un très grand nombre de tours autour de $\mathbf{F}$, de sorte qu'en valeur moyenne, $\mathbf{I}$ et $\mathbf{J}$ se réduisent à leur projection $\mathbf{I}_{\|}$et $\mathbf{J}_{\|}$sur $\mathbf{F}$ (voir Fig. 1). Ces deux projections ont pour longueur $|\mathbf{I}| \cos \theta / 2$ (où $\theta$ est l'angle entre $O z$ et $\mathbf{J}$ ). Il reste maintenant à faire les moyennes de $\mathbf{I}_{\|}$et $\mathbf{J}_{\|}$sur toutes les directions de $\mathbf{J}$ qui sont également probables. Par symétrie autour de l'axe $O z$, ces valeurs moyennes sont parallèles à cet axe : leur longueur s'obtient par une seconde projection sur $O z$, suivie d'une intégration, ce qui donne :

$$
\begin{aligned}
\left\langle I_{z}\right\rangle_{s} & =\left\langle J_{z}\right\rangle_{s}=\frac{1}{4 \pi} \int_{0}^{2 \pi} \mathrm{d} \varphi \int_{0}^{\pi} \cos ^{2} \frac{\theta}{2} \sin \theta \mathrm{d} \theta|\mathbf{I}| \\
& =\frac{1}{2}|\mathbf{I}| .
\end{aligned}
$$

On voit donc que, lorsque les atomes quittent le niveau par émission spontanée, la moitié de leur orientation nucléaire s'est transformée en orientation électronique.

Un raisonnement aussi simple que celui qui est exposé ci-dessus ne permet pas de calculer $\left\langle J_{z}\right\rangle_{s}$ dans le cas général où le produit $a \tau$, ainsi que le nombre quantique $J$, sont quelconques. Il est alors préférable, pour calculer $\left\langle J_{z}\right\rangle_{s}$, d'introduire la matrice densité $\rho$ des atomes dans l'état excité, et de calculer son évolution.

\section{Etude de la matrice densité ATOMiQUe. -}

a) Equation d'évolution de $\rho$. - Nous appelons $\rho$ la matrice densité des atomes qui se trouvent dans un état excité donné. Pour simplifier, nous nous limitons au cas où cet état est un niveau de structure fine, dont le nombre quantique $J$ est donné (ce « multiplet» se décompose, lorsque $J \neq 0$ et $I \neq 0$, en plusieurs sous-niveaux hyperfins $F$, et éventuellement en sousniveaux Zeeman). Nous supposons que le nombre quantique $I$ vaut $\frac{1}{2}$ (ce qui est le cas pour ${ }^{3} \mathrm{He}$ ) ; $F$ peut donc prendre deux valeurs $J \pm \frac{1}{2}$. Nous allons tenir compte de trois causes d'évolution de la matrice densité $\rho$ :

- Le peuplement de l'état excité sous l'effet des collisions que subissent les atomes dans la décharge ; nous considérons comme instantané ce processus d'excitation (cette hypothèse est liée à la très faible valeur du temps de collision $\tau_{\mathrm{c}}$, et donc à la grande dispersion en énergie des particules qui excitent l'atome).

- L'évolution propre dans l'état excité, sous l'influence d'un hamiltonien $H$.

- L'émission spontanée qui fait retomber les atomes dans des états d'énergies plus faibles. Ce processus peut aussi être considéré comme instantané (les phénomènes transitoires qui lui sont associés durent un temps de l'ordre de l'inverse de la fréquence optique associée à la transition).

Dans ces conditions, l'équation d'évolution de la matrice densité peut s'écrire :

$$
\frac{\mathrm{d}}{\mathrm{d} t} \rho=\frac{1}{T_{\mathrm{d}}} \rho^{0}+\frac{1}{i \hbar}[H, \rho]-\Gamma \rho
$$

où $1 / T_{\mathrm{d}}$ est proportionnel au nombre d'atomes que la décharge porte dans l'état excité par unité de temps ; $\rho^{0}$ est la matrice densité d'un atome immédiatement après excitation. On a posé $\Gamma=1 / \tau$.

Lorsque $\rho^{0}$ et $H$ sont indépendants du temps, on peut trouver la solution stationnaire de (I, A, 2) en écrivant :

$$
\frac{1}{T_{\mathrm{d}}} \rho^{0}+\frac{1}{i \hbar}[H, \rho]-\Gamma \rho=0 .
$$

Cette équation permet de calculer les éléments de matrice de $\rho^{s}$ en fonction de ceux de $\rho^{0}$; en effet, si l'on appelle $\mid n>$ les états propres de $H$, d'énergies $\hbar \omega_{n}$, on a :

$$
\left.<n\left|\rho^{s}\right| n^{\prime}\right\rangle=\frac{1}{T_{\mathrm{d}}} \frac{\left\langle n\left|\rho^{0}\right| n^{\prime}\right\rangle}{\Gamma+i\left(\omega_{n}-\omega_{n^{\prime}}\right)} .
$$

Lorsque $\rho^{0}$ est diagonale dans la base des états $|n\rangle, \rho^{s}$ est proportionnelle à $\rho^{0}$ :

$$
\rho^{s}=\frac{1}{\Gamma T_{\mathrm{d}}} \rho^{0}
$$

de sorte que l'évolution propre dans l'état excité n'a pas d'influence. Au contraire, si $\rho^{0}$ possède des éléments non diagonaux ou encore " cohérences » (cas d'une excitation « cohérente » [7]), $\rho^{s}$ n'est en général plus proportionnelle à $\rho^{0}$. Pour aller plus loin, nous devons donc connaître plus en détail la forme de $\rho^{0}$. 
b) Forme de $\rho^{0}$. $-\rho^{0}$ possède les deux propriétés suivantes :

- La matrice densité nucléaire correspond à une orientation du spin nucléaire $\mathbf{I}$, parallèle à un axe $O z$. Rappelons que nous supposons dans cet article que le nombre quantique $I$ vaut $\frac{1}{2}$; la matrice densité $\rho_{n}^{0}$, trace partielle de $\rho^{0}$ sur les variables électroniques, s'écrit donc $\left({ }^{4}\right)$ :

$$
\rho_{n}^{0}=\operatorname{Tr}_{\mathrm{e}}\left(\rho^{0}\right)=\frac{1}{2}+P I_{z}
$$

où $P$ est la polarisation nucléaire dans l'état fondamental $(0 \leqslant P \leqslant 1)$.

- $\rho^{0}$ est invariante lorsque l'on effectue une rotation sur les électrons seuls. La matrice densité électronique $\rho_{\mathrm{e}}^{0}$ est donc proportionnelle à la matrice unité :

$$
\rho_{\mathrm{e}}^{0}=\operatorname{Tr}_{n}\left(\rho^{0}\right)=\frac{1}{2 J+1} .
$$

Les égalités (I.A.5) et (I.A.6) ne suffisent pas à elles seules à déterminer $\rho^{0}$; il existe en effet une infinité de matrices ayant les mêmes traces partielles $\rho_{n}^{0}$ et $\rho_{\mathrm{e}}^{0}$, la plus simple étant la matrice obtenue par produit tensoriel :

$$
\rho^{0}=\rho_{n}^{0} \otimes \rho_{\mathrm{e}}^{0} .
$$

Il est possible de montrer que $\rho^{0}$ a effectivement cette forme simple, qui correspond au cas où il n'existe aucune corrélation entre les variables nucléaires et électroniques. En effet, nous avons supposé que le spin nucléaire n'est pas affecté lors de la collision qui excite l'atome ; cela signifie que les variables nucléaires ne dépendent que du cycle de pompage optique subi antérieurement par les atomes, c'est-à-dire de l' " histoire » des atomes avant leur excitation. Au contraire, les variables électroniques des atomes qui viennent d'être excités sont complètement indépendantes de cette « histoire »; en effet, le moment angulaire électronique dans l'état fondamental est nul, par hypothèse, et le moment angulaire $\mathbf{J}$ qui apparaît lors de l'excitation ne dépend que des caractéristiques diverses de la collision, et non des propriétés du spin nucléaire. Il n'existe donc aucune corrélation entre les variables nucléaires et électroniques $\left({ }^{5}\right)$, et $\rho^{0}$ a donc bien la forme (I.A.7).

Cette expression nous montre que $\rho^{0}$ est diagonale en représentation $\mid m_{I} m_{J}>$ (vecteur propre commun à

(4) Dans tout cet article, $\mathbf{I}$ et $\mathbf{J}$ désignent les moments angulaires nucléaire et électronique divisés par $\hbar$; avec cette convention, souvent utilisée, $\mathbf{I}$ et $\mathbf{J}$ sont des opérateurs vectoriels sans dimension.

(5) Cette absence de corrélation est présentée ici comme une conséquence du fait que la collision excitatrice est très rapide, propriété fondamentale de la méthode étudiée dans cet article. Il aurait aussi été possible de démontrer la relation (I.A.7) en utilisant l'invariance de $\rho^{0}$ par toute rotation portant sur les électrons seuls; on comprend en effet qu'il ne puisse exister aucune corrélation entre l'orientation de $I$ et une atmosphère électronique parfaitement isotrope.
$I_{z}$ et $J_{z}$ de valeurs propres respectives $m_{I}$ et $m_{J}$ ). Toutefois, ce ne sont en général pas ces vecteurs qui sont vecteurs propres de $H$; par exemple, en champ magnétique nul, ce sont les sous-niveaux hyperfins $\left|F, m_{F}\right\rangle$ (vecteurs propres communs à $\mathbf{F}^{2}$ et $F_{z}$, de valeurs propres $F(F+1)$ et $\left.m_{F}\right)$. La matrice $\rho^{0}$ possède des « cohérences » en représentation $\left|F, m_{F}\right\rangle$; d'après ce qui précède, il s'ensuit que $\rho^{s}$ n'est en général pas proportionnelle à $\rho^{0}$.

c) Calcul de $\rho^{s}$. Polarisation de la lumière émise par les atomes. - La relation (I.A.4) permet de calculer $\rho^{s}$ en fonction de $\rho^{0}$, qui est connue. On peut alors connaître l'intensité lumineuse $L\left(\mathbf{e}_{\lambda}\right)$ émise par les atomes dans une direction donnée $O z$ et avec une polarisation" $\mathbf{e}_{\lambda}$ grâce à la relation :

$$
\begin{aligned}
L\left(\mathbf{e}_{\lambda}\right)=\alpha \Gamma & \sum_{n n^{\prime}}\left\langle n\left|\rho^{s}\right| n^{\prime}\right\rangle \times \\
& \left.<n^{\prime}\left|\left(\mathbf{e}_{\lambda} . \mathbf{D}\right) P_{1}\left(\mathbf{e}_{\lambda}^{*} . \mathbf{D}\right)\right| n\right\rangle
\end{aligned}
$$

où $\alpha$ est une constante, $\mathbf{D}$ un opérateur vectoriel sans dimension proportionnel à l'opérateur dipôle électrique, et $P_{1}$ le projecteur sur les états d'énergies plus basses vers lesquels s'effectue la transition optique.

Supposons par exemple que l'hamiltonien dans l'état excité se réduise à un terme dû au couplage hyperfin $(H=\hbar a \mathbf{I} . \mathbf{J})$, et posons $\Delta W=a\left(J+\frac{1}{2}\right)(\hbar \Delta W$ est la structure hyperfine du niveau considéré). Calculons alors $\rho^{s}$; nous obtenons :

$$
\begin{aligned}
& <F, m_{F}\left|\rho^{s}\right| F, m_{F}^{\prime}>= \\
& \quad=\frac{1}{\Gamma T_{\mathrm{d}}}<F, m_{F}\left|\rho^{0}\right| F, m_{F}^{\prime}>
\end{aligned}
$$

et, lorsque $F \neq F^{\prime}$ :

$$
\begin{aligned}
& <F, m_{F}\left|\rho^{s}\right| F^{\prime}, m_{F}^{\prime}>= \\
& \quad=\frac{1}{T_{\mathrm{d}}(\Gamma \pm i \Delta W)}<F, m_{F}\left|\rho^{0}\right| F^{\prime}, m_{F}^{\prime}>.
\end{aligned}
$$

On peut alors distinguer deux cas extrêmes :

- Lorsque la structure hyperfine de l'état excité n'est pas résolue $(\Delta W \ll \Gamma)$, on peut négliger $\Delta W$ devant $\Gamma$ dans (I.A.10) et l'on obtient :

$$
\rho^{s} \simeq \frac{1}{\Gamma T_{\mathrm{d}}} \rho^{0} .
$$

La matrice $\rho^{s}$ possède alors, comme $\rho^{0}$, des cohérences hyperfines; elle correspond donc elle aussi à une orientation de $\mathbf{I}$ mais non de $\mathbf{J}$. Nous avons vu que dans ce cas la lumière émise par les atomes n'est pas polarisée, si l'on considère l'ensemble des composantes hyperfines de la raie spectrale étudiée (cf. note $\left({ }^{2}\right)$ ); c'est donc que, dans la formule (I.A.8), les effets des différences de populations et des cohérences de $\rho^{s}$ s'annulent entre eux, de sorte que $L\left(\mathbf{e}_{\lambda}\right)$ ne dépend pas de $\mathbf{e}_{\lambda}$.

- Au contraire, lorsque la structure hyperfine est complètement résolue dans l'état excité $(\Delta W \gg \Gamma)$, on 
voit sur (I.A.10) que les cohérences hyperfines de la matrice densité s'annulent au cours de l'évolution propre dans l'état excité. Lorsque l'atome retombe par émission spontanée dans un niveau d'énergie plus basse, l'effet de ces cohérences sur la polarisation de la lumière émise par les atomes disparaît et ne peut donc plus compenser celui des différences de population : la lumière émise est polarisée $\left({ }^{6}\right)$.

Considérons pour fixer les idées, le cas où $J$ vaut 1 (rappelons que $I=\frac{1}{2}$ ), et où la polarisation nucléaire $P$ de l'état fondamental vaut 1 (la direction de cette polarisation définit l'axe $O z$ de quantification). La relation (I.A.8) permet de calculer aisément $\rho^{0}$, dans la base des vecteurs propres $\left|F, m_{F}\right\rangle$ de $H$; on obtient :

$\left.m_{F}: \quad \begin{array}{cccc|cc}3 / 2 & 1 / 2 & -1 / 2 & -3 / 2 & \multicolumn{1}{c}{\frac{1 / 2}{1 / 2}} & -1 / 2 \\ 1 & \cdot & \cdot & \cdot & \dot{5} & \cdot \\ \cdot & 2 / 3 & \cdot & \cdot & \sqrt{2} / 3 & \dot{\sqrt{2}} / 3 \\ \cdot & \cdot & 1 / 3 & \cdot & \cdot & \cdot \\ \cdot & \cdot & \cdot & 0 & \cdot & \cdot \\ \hline \cdot & \sqrt{2} / 3 & \cdot & \cdot & 1 / 3 & \cdot \\ \cdot & \cdot & \sqrt{2} / 3 & \cdot & \cdot & 2 / 3\end{array}\right)$

(seuls sont donnés les éléments de matrice de $\rho^{0}$ non nuls). On voit que (et on montre que ces propriétés sont vraies quel que soit $J$ ) :

- $\rho^{0}$ ne possède que des cohérences hyperfines et pas de cohérences Zeeman ;

- il n'y a pas de différence de populations hyperfines : $\langle\mathbf{I} . \mathbf{J}\rangle_{0}=\operatorname{Tr}\left(\rho^{0} \mathbf{I} . \mathbf{J}\right)=0$.

- à l'intérieur des deux sous-niveaux hyperfins $F=\frac{1}{2}$ et $F=\frac{3}{2}$, on obtient deux orientations longitudinales et de signes opposés. On peut comprendre ce changement de signe en remarquant que, lorsque

$$
J>I=\frac{1}{2},
$$

I est parallèle à $\mathbf{F}$ dans le niveau $F=J+\frac{1}{2}$, antiparallèle dans le niveau $F=J-\frac{1}{2}$ (voir Fig. 2); on trouve bien ainsi une orientation positive dans le premier sous-niveau hyperfin, négative dans le second.

Lorsque l'on a $\Delta W \gg \Gamma$, nous avons vu que $\rho^{s}$ s'obtient en divisant $\rho^{0}$ par $\Gamma T_{\mathrm{d}}$ et en supprimant les

(6) Ce phénomène peut être rapproché de l'effet de dépolarisation magnétique dit ((effet Hanle ) [14], [7]. On sait que cet effet se produit lorsqu'une excitation lumineuse de polarisation ( cohérente) introduit dans le niveau excité des ( cohérences Zeeman ». Ces dernières subsistent ou disparaissent au cours de l'évolution propre dans l'état excité, suivant la valeur du champ magnétique statique $\mathbf{B}_{0}$ appliqué, de sorte que la polarisation de la lumière émise par les atomes dépend de $B_{0}$. Notons toutefois que, dans le cas de l'effet Hanle, la disparition des cohérences entraîne une dépolarisation de la lumière, alors que dans le processus étudié dans cet article, c'est au contraire cette disparition des cohérences qui est responsable de l'apparition de la polarisation lumineuse.

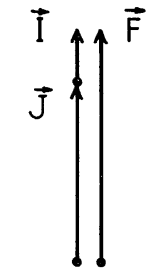

$\mathrm{F}=\mathrm{J}+1 / 2$

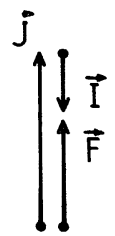

$F=J-1 / 2$
FIg. 2. - Position relative de $\mathbf{I}, \mathbf{J}$ et $\boldsymbol{F}$ dans les deux niveaux hyperfins $F=J-\frac{1}{2}$ et $F=J+\frac{1}{2}$.

« cohérences hyperfines ». Cette opération effectuée, calculons les intensités lumineuses $L\left(\sigma_{+}\right)$et $L\left(\sigma_{-}\right)$ en utilisant les "diagrammes de Grotrian » donnant les carrés des éléments de matrice de D (cf. Fig. 3), pour une transition optique $J=1 \rightarrow J=0$; nous obtenons :

$$
\left.\begin{array}{l}
\text { composante } F=3 / 2: L_{F}\left(\sigma_{+}\right) \propto 11 / 9 \quad L_{F}\left(\sigma_{-}\right) \propto 1 / 9 \\
\text { composante } F=1 / 2: L_{F}\left(\sigma_{+}\right) \propto 2 / 9 \quad L_{F}\left(\sigma_{-}\right) \propto 4 / 9 \\
\mathrm{~m}_{\mathrm{F}}:-\frac{3}{2} \quad-\frac{1}{2} \quad+\frac{1}{2} \quad+\frac{3}{2} \\
\mathrm{~F}=\frac{1}{2}
\end{array}\right\}
$$

FIG. 3. - Diagramme donnant les probabilités relatives des différentes transitions optiques d'un niveau $J=1$ vers un niveau $J=0$, en tenant compte de la structure hyperfine due au spin nucléaire $\Gamma=\frac{1}{2}$.

Les deux composantes hyperfines $F=3 / 2$ et $F=1 / 2$ sont donc polarisées circulairement et en sens inverse ; pour l'ensemble des composantes hyperfines, on obtient :

$$
L_{F}\left(\sigma_{+}\right) \propto 13 / 9 ; \quad L_{F}\left(\sigma_{-}\right) \propto 5 / 9
$$

soit un taux de polarisation égal à :

$$
\frac{L_{F}\left(\sigma_{+}\right)-L_{F}\left(\sigma_{-}\right)}{L_{F}\left(\sigma_{+}\right)+L_{F}\left(\sigma_{-}\right)}=\frac{8}{18} \simeq 44 \% .
$$

La lumière émise par les atomes est donc, dans ce cas, fortement polarisée.

d) Influence d'un champ magnétique statique appli$q u e$. - Nous avons supposé jusqu'ici que l'évolution des atomes dans l'état excité est due à la seule influence du couplage hyperfin $a \mathbf{I} . \mathbf{J}$. On peut se 
demander ce qui se produit lorsque l'on place les atomes dans un champ magnétique statique $\mathbf{B}_{0}$ qui introduit un nouveau terme dans l'hamiltonien $\boldsymbol{H}$. Dans ce paragraphe, nous nous contenterons d'une étude qualitative, repoussant le calcul détaillé de l'effet de $\mathbf{B}_{0}$ au paragraphe suivant. On peut distinguer trois cas :

* Champ magnétique faible. - Ce cas est celui où $\mathbf{B}_{0}$ est trop faible pour que la précession de Larmor puisse faire tourner $\mathbf{I}$ ou $\mathbf{J}$ d'une fraction notable de tour, pendant la durée de vie $\tau$; on peut alors négliger l'influence du champ magnétique $\left({ }^{7}\right)$, de sorte que ce cas correspond à celui qui a déjà été étudié. Nous avons vu qu'alors il peut apparaître une orientation électronique $\langle\mathbf{J}\rangle_{s}$ dans l'état excité. Remarquons cependant que les diverses composantes de l'alignement électronique restent constamment nulles car l'hamiltonien hyperfin, invariant par rotation, ne peut coupler orientation et alignement (grandeurs tensorielles d'ordre différent). La lumière émise par les atomes possède donc un certain taux de polarisation circulaire, mais aucune polarisation linéaire.

* Champ magnétique fort. - I et $\mathbf{J}$ sont totalement découplés; au cours de l'évolution propre dans l'état excité, aucune orientation électronique n'apparaît.

* Champ magnétique intermédiaire. - I et $\mathbf{J}$ sont partiellement découplés. On doit alors tenir compte à la fois de l'existence du champ magnétique et du découplage hyperfin. Comme plus haut, ce dernier peut transférer l'orientation de $\mathbf{I}$ à $\mathbf{J}$. Cependant dans ce cas, l'hamiltonien n'est plus invariant par rotation (il n'est invariant que lorsque l'axe de la rotation est parallèle à $\mathbf{B}_{0}$ ), de sorte qu'un couplage entre l'orientation et l'alignement n'est plus exclus. On comprend aisément que le champ magnétique, qui tend à faire précesser $\mathbf{I}$ et $\mathbf{J}$ avec des vitesses angulaires différentes, modifie leur position relative; le système formé par ces deux vecteurs est alors déformé, et il est possible qu'au cours de l'évolution propre dans l'état excité, il apparaisse un alignement. On peut donc avoir à la fois $\left\langle J_{z}\right\rangle_{s} \neq 0$ et $\left\langle 3 J_{z}^{2}-J(J+1)\right\rangle_{s} \neq 0$ : la lumière émise par les atomes peut alors posséder à la fois un taux de polarisation circulaire et linéaire.

(7) Notons toutefois que l'effet de $\mathbf{B}_{0}$, s'il est négligeable dans l'état excité, peut être important dans l'état fondamental, dont l'orientation nucléaire subit la précession de Larmor avec la pulsation $\omega_{f}$. Il s'ensuit que, lorsque l'orientation de l'état fondamental est transversale, $\rho^{0}$ dépend du temps; les équations (I.A.3) et (I.A.4) ne sont alors en général plus valables. On peut toutefois montrer que, lorsque les constantes de temps de l'état fondamental sont très longues devant celles de l'état excité (condition $\omega_{f} \ll \Gamma$ ), ce dernier peut suivre de façon ( quasi instantanée ) le mouvement de l'état fondamental; les équations (I. A. 3) et (I. A.4) constituent alors une bonne approximation, où $\rho^{s}$ est une fonction oscillante du temps, de pulsation $\omega_{f}$. Dans ce cas, l'orientation électronique $\left\langle J_{z}\right\rangle_{s} d e$ l'état excité est modulée à la fréquence $\omega_{f} / 2 \pi$ de l'état fondamental; des expériences où ce phénomène a été mis en évidence ont été réalisées [15], [16]
B. Etude précise ; calcul de l'orientation et de l'alignement électroniques ; polarisation des raies lumineuses émises. - Dans le paragraphe précédent, nous nous sommes limités à l'étude de quelques cas particuliers $\left(J=\frac{1}{2}\right.$ ou $\left.1, B_{0}=0, \Delta W . \tau \gg 1\right)$. Nous nous proposons, dans ce paragraphe, de calculer de manière générale l'orientation et l'alignement électroniques, et d'en déduire les divers taux de polarisation circulaire ou linéaire de la lumière émise par les atomes, lorsque le spin nucléaire I est orienté dans l'état fondamental.

1. Hypothèses. Principe du Calcul. - Nous supposons que l'on s'intéresse à une composante de structure fine d'une des raies spectrales émises par les atomes. Nous nous limitons donc à l'étude de la matrice densité $\rho$ d'un «multiplet » de moment cinétique $J$ donné ; nous excluons donc le cas où $J$ n'est pas un «bon nombre quantique » (cas qui pourrait par exemple se produire en champ magnétique $\mathbf{B}_{0}$ très intense, ou pour un niveau dont la structure hyperfine serait du même ordre de grandeur que la structure fine $\left({ }^{8}\right)$ ).

La transition optique correspondant à la raie spectrale étudiée s'effectue entre le niveau de moment angulaire $J$ et un niveau d'énergie inférieure et de moment angulaire électronique $J_{1}$ (cf. Fig. 4). Les valeurs de $J$ et de $J_{1}$ peuvent être quelconques; par contre, nous conservons l'hypothèse $I=\frac{1}{2}$. Nous ne faisons aucune hypothèse concernant les valeurs relatives de $\tau$ (durée de vie), $\hbar \Delta W$ (structure hyperfine) et $B_{0}$ (champ magnétique statique) ; par exemple, un découplage hyperfin partiel ou total n'est pas exclu. Enfin, nous supposons que l'orientation nucléaire de l'état fondamental est longitudinale, c'est-à-dire parallèle à l'axe $O z$ qui porte $\mathbf{B}_{0}$.

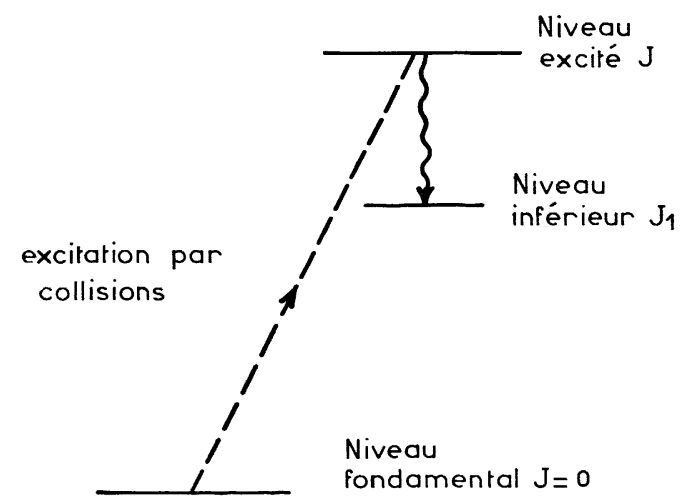

Fig. 4. - Niveaux intervenant dans l'excitation des atomes et dans la détection optique.

Calculons $L\left(\mathbf{e}_{\lambda}\right)$, intensité de la lumière émise par les atomes dans une direction $\mathrm{Oz}$, avec une polarisation $\mathbf{e}_{\lambda}$, et dont la longueur d'onde correspond à la

(8) Le cas se produit par exemple pour certains niveaux triplets de ${ }^{3} \mathrm{He}$, dont la structure hyperfine est très importante. 
transition du niveau $J$ au niveau $J_{1}$. En regroupant les formules (I.A.4) et (I.A.8), nous obtenons :

$$
\begin{aligned}
L\left(\mathbf{e}_{\lambda}\right) & =\alpha \Gamma \operatorname{Tr}\left\{\rho^{s} A\left(\mathbf{e}_{\lambda}\right)\right\}=\alpha \Gamma<A\left(\mathbf{e}_{\lambda}\right)>_{s} \\
& =\alpha \Gamma \sum_{n, n^{\prime}}<n\left|\rho^{0}\right| n^{\prime}> \\
& \frac{1}{\Gamma+i\left(\omega_{n}-\omega_{n^{\prime}}\right)}<n^{\prime}\left|A\left(\mathbf{e}_{\lambda}\right)\right| n>
\end{aligned}
$$

avec

$$
A\left(\mathbf{e}_{\lambda}\right)=\left(\mathbf{e}_{\lambda} . \mathbf{D}\right) P_{1}\left(\mathbf{e}_{\lambda}^{*} . \mathbf{D}\right) .
$$

Rappelons que $P_{1}$ est le projecteur sur les états atomiques correspondant au niveau inférieur $\left(J=J_{1}\right)$ de la transition optique. On voit que, dans l'expression (I.B.1), interviennent trois facteurs :

$\left.-<n\left|\rho^{0}\right| n^{\prime}\right\rangle$, qui rend compte de l'excitation des atomes dans le niveau étudié. Ce facteur dépend implicitement de l'hamiltonien $H$ : lorsque $H$ varie (par exemple si $B_{0}$ varie), les états $|n\rangle$ et $\left|n^{\prime}\right\rangle$ changent et l'élément de matrice $\left\langle n\left|\rho^{0}\right| n^{\prime}\right\rangle$ est modifié.

$\left.-<n^{\prime}\left|A\left(\mathbf{e}_{\lambda}\right)\right| n\right\rangle$, qui est l'élément de matrice d'un opérateur correspondant à la détection lumineuse. Comme le précédent, ce facteur dépend de $H$.

$-\left[\Gamma+i\left(\omega_{n}-\omega_{n^{\prime}}\right)\right]^{-1}$ qui ne dépend que des énergies des vecteurs propres de $H$. Ce facteur est résonnant lorsque $\omega_{n}=\omega_{n^{\prime}}$; c'est lui qui est responsable des effets dits de "croisements de niveaux ".

On peut montrer que $A\left(\mathbf{e}_{\lambda}\right)$ est un opérateur qui, comme D, n'agit que dans l'espace des variables électroniques, et laisse invariant le spin nucléaire I. En effet, nous avons supposé que $L\left(\mathbf{e}_{\lambda}\right)$ est la lumière émise par les atomes sur l'ensemble des composantes hyperfines d'une raie spectrale : $P_{1}$ est donc le projecteur sur l'ensemble des sous-niveaux hyperfins et Zeeman du niveau inférieur de $J_{1} ; P_{1}$ n'agit donc pas sur I ; on voit alors sur l'expression (I.B.2) que $A\left(\mathbf{e}_{\lambda}\right)$ possède la même propriété. Cela entraîne que, lorsque $\rho^{s}$ ne correspond qu'à une orientation nucléaire $\left(\rho^{s}\right.$ reste donc invariante par rotation des électrons seuls), $\left\langle A\left(\mathbf{e}_{\lambda}\right)\right\rangle_{s}$, invariant par rotation de $\mathbf{e}_{\lambda}$, ne dépend pas de $\mathbf{e}_{\lambda}$. Nous établissons ainsi un résultat qui a été utilisé plus haut (cf. note 2) :

$L\left(\mathbf{e}_{\lambda}\right)$ ne dépend que des grandeurs électroniques, et non des variables nucléaires.

Pour préciser ces grandeurs électroniques, il est utile de remarquer que $P_{1}$ est scalaire ; ceci entraîne, D étant vectoriel, que la décomposition de $A\left(\mathbf{e}_{\lambda}\right)$ sur des opérateurs tensoriels ${ }^{J} T_{q}^{(k)}$ ne fait intervenir que les ordres 0,1 et 2 . L'invariance par rotation autour de $O z$ du problème étudié entraîne une autre simplification : les seules valeurs moyennes $\left\langle{ }^{J} T_{q}^{(k)}\right\rangle_{s}$ non nulles satisfont la relation $q=0$. En conclusion, l'intensité et la polarisation de la lumière émise par les atomes ne peuvent dépendre que de trois quantités :

- la population totale de l'état excité,

- l'orientation électronique $\left\langle J_{z}\right\rangle_{s}$,

- l'alignement électronique $\left\langle 3 J_{z}^{2}-J(J+1)\right\rangle_{s}$.
Aussi nous allons calculer ces trois valeurs moyennes dans le paragraphe suivant.

2. CALCUl DE $\left\langle J_{z}\right\rangle_{s}$ ET $\left\langle J_{z}^{2}\right\rangle_{s^{-}}-$a) Notation; niveaux d'énergie. - L'hamiltonien $H$ dans l'état excité s'écrit, avec les conventions de la note $\left({ }^{4}\right)$ :

$$
H=-g_{J} \mu_{\mathrm{B}} B_{0} J_{z}-g_{n} \mu_{n} B_{0} I_{z}+\hbar a \mathbf{I} . \mathbf{J} \text { (I.B.3) }
$$

où $\mu_{\mathrm{B}}=q \hbar / 2 m$ est le magnéton de Bohr; $g_{J}$ le facteur de Landé du niveau excité ; $m_{I} g_{n} \mu_{n}$ (où $m_{I}=\frac{1}{2}$ ) est le moment magnétique du noyau. Rappelons que $a$ est la constante de couplage hyperfin (exprimée en $\mathrm{rd} / \mathrm{s})$ à l'intérieur du niveau considéré.

La recherche des valeurs propres et des vecteurs propres de $H$ est un problème classique; on utilise, pour le résoudre, le fait que $H$ commute avec $F_{z}=I_{z}+J_{z}$, ce qui permet de ramener le problème à la diagonalisation de sous-matrices $2 \times 2$, correspondant à la restriction de $H$ au sous-espace propre de $F_{z}$ de valeur propre $m_{F}$ donnée ; en appelant $P_{m_{F}}$ le projecteur sur ce sous-espace, on a, dans la base des deux vecteurs $\mid m_{I}=\frac{1}{2}, m_{J}=m_{F}-\frac{1}{2}>$ et

$$
\mid m_{I}=-\frac{1}{2}, m_{J}=m_{F}+\frac{1}{2}>,
$$

pris dans cet ordre :

$$
\begin{aligned}
P_{m_{F}} H P_{m_{F}} & =-g_{J} \mu_{\mathrm{B}} B_{0} m_{F}-\frac{\hbar a}{4}+ \\
& +\frac{\hbar}{2}\left(\alpha B_{0}+a m_{F}\right) \sigma_{3}+\frac{\hbar}{2} \beta\left(m_{F}\right) \sigma_{1}
\end{aligned}
$$

où l'on a posé :

$$
\begin{aligned}
& \hbar \alpha=g_{J} \mu_{\mathrm{B}}-g_{n} \mu_{n} \simeq g_{J} \mu_{\mathrm{B}} \\
& \beta\left(m_{F}\right)=a \sqrt{(J+1 / 2)^{2}-m_{F}^{2}}
\end{aligned}
$$

$\sigma_{1}, \sigma_{2}$ et $\sigma_{3}$ désignent les trois matrices de Pauli.

Introduisons l'angle $\varphi\left(m_{F}\right)$ par la relation :

$$
\operatorname{cotg} 2 \varphi=\frac{\alpha B_{0}+a m_{F}}{\beta}
$$

avec $0 \leqslant \varphi \leqslant \pi / 2$.

Les deux vecteurs propres de $P_{m_{F}} H P_{m_{F}}$ s'expriment simplement en fonction de $\varphi$ dans la base des vecteurs propres $\mid m_{I} m_{J}>$ communs à $I_{z}$ et $J_{z}$ :

$$
\left\{\begin{aligned}
\mid m_{F}, 1>= & \cos \varphi\left|+\frac{1}{2}, m_{F}-\frac{1}{2}\right\rangle+ \\
& +\sin \varphi \mid-\frac{1}{2}, m_{F}+\frac{1}{2}> \\
\mid m_{F}, 2>= & \sin \varphi \mid+\frac{1}{2}, m_{F}-\frac{1}{2}>- \\
& -\cos \varphi \mid-\frac{1}{2}, m_{F}+\frac{1}{2}>
\end{aligned}\right.
$$

Les valeurs propres respectives de ces vecteurs sont $(j=1,2)$ :

$$
\hbar \omega_{F}^{j}=-\left[g_{J} \mu_{\mathrm{B}} B_{0} m_{F}+\frac{\hbar a}{4}+(-1)^{j} \frac{\hbar \beta}{2 \sin 2 \varphi}\right],
$$


(La phase des deux vecteurs $\mid m_{F}, 1>$ et $\mid m_{F}, 2>$ a été choisie de telle manière que, lorsque $B_{0}$ tend vers zéro, ces deux vecteurs tendent vers

$\mid I, J, F=J+\frac{1}{2}, m_{F}>$ et $\mid I, J, F=J-\frac{1}{2}, m_{F}>$ )

Lorsque $\alpha \boldsymbol{B}_{0}=-a m_{F}$, on a $\varphi\left(m_{F}\right)=\pi / 4$, et la différence d'énergie entre les deux niveaux est minimale, mais non nulle; on dit que les deux niveaux «s'anticroisent ». Toutes les valeurs de $m_{F}$,' à l'exception des valeurs extrêmes $m_{F}= \pm\left(J+\frac{1}{2}\right)$, donnent un tel anticroisement; ces derniers sont donc au nombre de $2 J$, disposés symétriquement par rapport à la valeur $B_{0}=0$.

b) Calcul. - Calculons la valeur moyenne :

$$
\begin{aligned}
<J_{z}>_{s}= & \operatorname{Tr}\left\{\rho^{s} J_{z}\right\} \\
= & \frac{1}{\Gamma T_{\mathrm{d}}} \sum_{m_{F}} \sum_{i, j=1,2}<m_{F}, i\left|\rho^{0}\right| m_{F}, j>\times \\
& \times \frac{\Gamma}{\Gamma+i\left(\omega_{m_{F}}^{i}-\omega_{m_{F}}^{j}\right)} \times \\
& \times<m_{F}, j\left|J_{z}\right| m_{F}, i>
\end{aligned}
$$

Il est donc nécessaire d'évaluer les éléments de matrice de $\rho^{0}$ et $J_{z}$; les relations (I.A.5), (I.A.6), (I.A.7) et (I.B.8) nous donnent :

$$
\begin{aligned}
& \left\langle m_{F}= \pm\left(J+\frac{1}{2}\right)\left|\rho^{0}\right| m_{F}= \pm\left(J+\frac{1}{2}\right)\right\rangle= \\
& =\frac{1}{4 J+2}(1 \pm P) \\
& <m_{F}, 1\left|\rho^{0}\right| m_{F}, 1>=\frac{1}{4 J+2}(1+P \cos 2 \varphi) \\
& <m_{F}, 1\left|\rho^{0}\right| m_{F}, 2>=\frac{1}{4 J+2} P \sin 2 \varphi \\
& <m_{F}, 2\left|\rho^{0}\right| m_{F}, 1>=\frac{1}{4 J+2} P \sin 2 \varphi \\
& <m_{F}, 2\left|\rho^{0}\right| m_{F}, 2>=\frac{1}{4 J+2}\left(1-P \cos 2^{-} \varphi\right) \\
& <m_{F}= \pm\left(J+\frac{1}{2}\right)\left|J_{z}\right| m_{F}= \pm\left(J+\frac{1}{2}\right)>= \pm J \\
& <m_{F}, 1\left|J_{z}\right| m_{F}, 1>=m_{F}-\frac{1}{2} \cos 2 \varphi \\
& \left\{<m_{F}, 1\left|J_{z}^{\bar{T}}\right| m_{F}, 2>=-\frac{1}{2} \sin 2 \varphi\right. \\
& <m_{F}, 2\left|\overrightarrow{J_{z}}\right| m_{F}, 1>=-\frac{1}{2} \sin 2 \varphi \\
& <m_{F}, 2\left|J_{z}\right| m_{F}, 2>=m_{F}+\frac{1}{2} \cos 2 \varphi \text {. }
\end{aligned}
$$

Reportons (I. B.9), (I. B. 11) et (I. B. 12) dans (I. B. 10); nous obtenons :

$$
\begin{aligned}
& <J_{z}>_{s}=\frac{1}{\Gamma T_{\mathrm{d}}(4 J+2)}\{J(1+P)-J(1-P)+ \\
& +\sum_{m_{F}=-J+1 / 2}^{J-1 / 2}\left(m_{F}-\frac{1}{2} \cos 2 \varphi\right)+\left(m_{F}+\frac{1}{2} \cos 2 \varphi\right) \\
& +P\left[\cos 2 \varphi\left(m_{F}-\frac{1}{2} \cos 2 \varphi\right)\right. \\
& -\cos 2 \varphi\left(m_{F}+\frac{1}{2} \cos 2 \varphi\right) \\
& +\frac{\Gamma}{\Gamma+(i \beta / \sin 2 \varphi)}\left(-\frac{1}{2} \sin ^{2} 2 \varphi\right) \\
& \left.\left.+\frac{\Gamma}{\Gamma-(i \beta / \sin 2 \varphi)}\left(-\frac{1}{2} \sin ^{2} 2 \varphi\right)\right]\right\}
\end{aligned}
$$

soit, tous calculs faits $\left({ }^{9}\right)$ :

$$
<J_{z}>_{s}=\frac{1}{\Gamma T_{\mathrm{d}}} \frac{P}{4 J+2} \sum_{m_{F}} f_{m_{F}}\left(B_{0}\right)
$$

avec :

$$
\begin{aligned}
f_{m_{F}}\left(B_{0}\right) & =\frac{\beta^{2}}{\Gamma^{2}+\beta^{2}\left(\sin ^{2} 2 \varphi\right)^{-1}} \\
& =\frac{a^{2}\left[\left(J+\frac{1}{2}\right)^{2}-m_{F}^{2}\right]}{\Gamma^{2}+a^{2}\left[\left(J+\frac{1}{2}\right)^{2}-m_{F}^{2}\right]+\left(\alpha B_{0}+a m_{F}\right)^{2}} .
\end{aligned}
$$

Le calcul de $\left\langle J_{z}^{2}\right\rangle_{s}=\operatorname{Tr}\left\{\rho^{s} J_{z}^{2}\right\}$ se fait de manière analogue, en utilisant les égalités :

$\left\{\begin{array}{l}<m_{F}= \pm\left(J+\frac{1}{2}\right)\left|J_{z}^{2}\right| m_{F}= \pm\left(J+\frac{1}{2}\right)>=J^{2} \\ <m_{F}, 1\left|J_{z}^{2}\right| m_{F}, 1>=m_{F}^{2}-m_{F} \cos 2 \varphi+\frac{1}{4} \\ <m_{F}, 1\left|J_{z}^{2}\right| m_{F}, 2>=-m_{F} \sin 2 \varphi \\ <m_{F}, 2\left|J_{z}^{2}\right| m_{F}, 1>=-m_{F} \sin 2 \varphi \\ <m_{F}, 2\left|J_{z}^{2}\right| m_{F}, 2>=m_{F}^{2}+m_{F} \cos 2 \varphi+\frac{1}{4}\end{array}\right.$

et l'on obtient :

$$
\begin{aligned}
<J_{z}^{2}>_{s}= & \frac{1}{\Gamma T_{\mathrm{d}}}\left\{\frac{J(J+1)}{3}+\right. \\
& \left.+\frac{2 P}{4 J+2} \sum_{m_{F}} m_{F} f_{m_{F}}\left(B_{0}\right)\right\} .
\end{aligned}
$$

(9) Dans le cas où $J=\frac{1}{2}, B_{0}=0$, et $a \gg \Gamma$, la formule (I.B.13) donne :

$$
<J_{z}>_{s}=<I_{z}>_{s}=\frac{1}{4} \frac{P}{\Gamma T_{\mathrm{d}}} .
$$

Une polarisation électronique de 1 correspondrait à

$$
<J_{z}>_{s}=J \times \operatorname{Tr} \rho_{s}=\frac{1}{2 \overline{\Gamma T_{\mathrm{d}}}}
$$

la polarisation électronique obtenue est donc égale à la moitié de la polarisation nucléaire $P$, et nous retrouvons bien le résultat établi au paragraphe I. A.1. 
Il est commode pour la suite de cet article d'introduire deux paramètres sans dimension :

$x=\alpha B_{0} / a$, qui rend compte du degré de découplage hyperfin dû au champ magnétique.

$\gamma=1 / a \tau=\Gamma / a$ qui dépend du rapport entre la largeur naturelle du niveau et sa structure hyperfine.

On a alors :

$m_{F}\left(B_{0}\right)=\frac{\left(J+\frac{1}{2}\right)^{2}-m_{F}^{2}}{\left(x+m_{F}\right)^{2}+\gamma^{2}+\left(J+\frac{1}{2}\right)^{2}-m_{F}^{2}}$.

Les équations (I.B.13) et (I.B.16) nous permettent de calculer l'orientation et l'alignement pour toute valeur de $a, \Gamma, B_{0}$ et $J$; elles appellent quelques remarques, qui sont exposées dans le paragraphe suivant.

c) Etude des résultats obtenus. - On voit sur (I.B.13) et (I.B.16) que, lorsque $P=0$, on a à la fois $\left\langle J_{z}\right\rangle_{s}=0$ et $\left\langle 3 J_{z}^{2}-J(J+1)\right\rangle_{s}=0$. L'orientation et l'alignement sont nuls lorsque l'état fondamental n'est pas orienté. De même, ces valeurs moyennes sont aussi nulles si $a=0$ [en effet dans ce cas, $\left.f_{m_{F}}\left(B_{0}\right)=0\right]$ : en l'absence de couplage hyperfin, l'orientation du niveau excité reste purement nucléaire.

Lorsque $a$ tend vers 0 , l'orientation et l'alignement électroniques tendent vers 0 comme des infiniment petits du second ordre par rapport à la quantité infinitésimale sans dimension $a \tau$. Le résultat est à rapprocher de ceux de Lehmann [5] concernant les variations de l'efficacité du pompage optique nucléaire de $\mathrm{Zn}$, $\mathrm{Cd}, \mathrm{Hg}$ en fonction de la constante $a$ du niveau de résonance optique.

La définition de la fonction $f_{m_{F}}\left(B_{0}\right)$ montre que

$$
f_{m_{F}}\left(B_{0}\right)=f_{-m_{F}}\left(-B_{0}\right) \text {; }
$$

$\left\langle J_{z}\right\rangle_{s}$ est donc une fonction paire de $B_{0}$, alors que $<3 J_{z}^{2}-J(J+1)>{ }_{s}$ est impaire.

En champ nul $(x=0)$, on obtient dans l'état excité une orientation de même signe que celle de l'état fondamental ; par contre, on n'obtient aucun alignement (cf. $\S$ I.A.2.d).

Lorsque $x$ tend vers l'infini, l'orientation et l'alignement tendent vers 0 . Nous retrouvons bien le fait que, lorsque I et $\mathbf{J}$ sont totalement découplés, l'orientation du niveau excité reste purement nucléaire.

Lorsque $x$ est de l'ordre de 1 (cas du découplage hyperfin partiel), on obtient non seulement une orientation, mais encore un alignement électronique. $\mathrm{Ce}$ dernier a un signe différent suivant que le champ magnétique $\mathbf{B}_{0}$ est parallèle ou antiparallèle à l'orientation nucléaire de l'état fondamental ; par contre ce signe ne dépend pas de $a$, qui n'intervient dans les équations que par son carré. Cette remarque explique pourquoi les expériences que nous avons réalisées ne permettent pas de déterminer le signe de $a$. Remarquons aussi que, si l'on avait $g_{J} \mu_{\mathrm{B}}=g_{n} \mu_{n}, x$ et donc l'alignement resteraient constamment nuls ; l'apparition de l'alignement est donc bien due au fait que $\mathbf{B}_{0}$ tend à faire tourner $\mathbf{I}$ et $\mathbf{J}$ à des vitesses angulaires différentes, et déforme ainsi le système formé par ces deux vecteurs (cf. $\S$ I.A.2.d).

Les variations de $f_{m_{F}}\left(B_{0}\right)$ en fonction de $B_{0}$ correspondent à une courbe de Lorentz, centrée en $x=-m_{F}$, soit $B_{0}=-a m_{F} / \alpha ;$ nous voyons que chaque fonction $f_{m_{F}}\left(B_{0}\right)$ correspond à la contribution de l'un des «anticroisements» de niveaux signalés plus haut (cf. $\S$ I.B.2.a). On pourrait penser que, pour ces valeurs de $B_{0}$ où la distance de deux niveaux qui « s'anticroisent » est minimale, et où les cohérences sont donc le moins détruites au cours de l'évolution propre, l'orientation électronique est minimale ; or c'est exactement le contraire qui se produit. C'est donc que, dans l'expression (I.B.10), les variations des éléments de matrice $\left.<m_{F}, i\left|\rho^{0}\right| m_{F}, j\right\rangle$ et $<m_{F}, j\left|J_{z}\right| m_{F}, i>$ compensent les variations $\mathrm{du}$ terme $\left[\Gamma+i\left(\omega_{F}^{i}-\omega_{F}^{j}\right)\right]^{-1}$, et donnent un maximum là où l'on aurait pu croire que se trouve un minimum.

3. Polarisation des Raies lumineuses Émises. Nous avons calculé dans le paragraphe précédent l'orientation et l'alignement électroniques, qui sont les observables dont dépend le taux de polarisation de la lumière émise par les atomes. Dans ce paragraphe, nous donnons le calcul explicite de $L\left(\mathbf{e}_{\lambda}\right)$ en fonction de $\left\langle J_{z}\right\rangle_{s}$ et $\left\langle J_{z}^{2}\right\rangle_{s}$; nous obtenons ainsi les expressions qui permettent de connaître théoriquement les signaux lumineux relatifs à une expérience donnée, en fonction des divers paramètres physiques (structure hyperfine, durée de vie, etc...). Le calcul est fait en deux étapes :

- dans la première, nous calculons $L\left(\mathbf{e}_{\lambda}\right)$ en fonction des valeurs moyennes $\left\langle{ }^{J} T_{q}^{(k)}\right\rangle_{s}=\operatorname{Tr}\left\{\rho^{s} T_{q}^{(k)}\right\}$ dans l'état excité de l'opérateur ${ }^{J} T_{q}^{(k)}$;

- dans la seconde, nous exprimons ces valeurs moyennes en fonction de $\left\langle J_{z}\right\rangle_{s}$ et de $\left\langle J_{z}^{2}\right\rangle_{s}$, ce qui permet d'obtenir le résultat final.

a) Décomposition de $A\left(\mathbf{e}_{\lambda}\right)$ en opérateurs tensoriels. - Il est classique d'utiliser des techniques de calculs basées sur l'utilisation des opérateurs tensoriels irréductibles pour étudier les expériences de pompage optique (voir par exemple les références [17] à [22]).

Soit $O Z$ la direction d'observation, $\left|\mathbf{e}_{X}\right\rangle$ et $\left|\mathbf{e}_{Y}\right\rangle$ deux vecteurs unitaires correspondant aux «vecteurs polarisation », parallèles aux axes $O X$ et $O Y$ d'un trièdre trirectangle $O X Y Z$; on peut poser :

$\left|\mathbf{e}_{\lambda}\right\rangle=\mathrm{e}^{-i \theta} \cos \psi\left|\mathbf{e}_{X}>+\mathrm{e}^{i \theta} \sin \psi\right| \mathbf{e}_{Y}>$ (I. B. 18) $\theta$ et $\psi$ sont deux paramètres qui caractérisent $\mathbf{e}_{\lambda}$. Les opérateurs tensoriels ${ }^{J} T_{q}^{(k)}$ sont définis par :

${ }^{J} T_{q}^{(k)}=\sum_{q^{\prime}+q^{\prime \prime}=q}<11 q^{\prime} q^{\prime \prime} \mid k q>D_{q^{\prime}} P_{1} D_{q^{\prime \prime}}$

( $D_{q}$ composante standard de $D$ dans les axes $O X Y Z$ ).

Remarquons que ${ }^{J} T_{q}^{(k)}$ dépend, par l'intermédiaire de $P_{1}$, du niveau inférieur $J_{1}$ de la transition optique. Avec ces définitions, on obtient par un calcul ana- 
logue $\left({ }^{10}\right)$ à celui qui donne l'équation (III.13) de la référence [22] :

$$
\begin{aligned}
L\left(\mathbf{e}_{\lambda}\right)=\alpha \Gamma\left\{\frac{1}{2}(\cos 2 \psi-i \sin 2 \psi \cos 2 \theta)<{ }^{J} T_{2}^{(2)}\right\rangle_{s}+ \\
\left.+\frac{1}{2}(\cos 2 \psi+i \sin 2 \psi \cos 2 \theta)<{ }^{J} \boldsymbol{T}_{-2}^{(2)}\right\rangle_{s} \\
-\frac{1}{\sqrt{6}}\left(<{ }^{J} T_{0}^{(2)}>_{s}+\sqrt{2}<{ }^{J} T_{0}^{(0)}>_{s}\right)- \\
\left.-\frac{1}{\sqrt{2}} \sin 2 \psi \sin 2 \theta<{ }^{J} \boldsymbol{T}_{0}^{(1)}>_{s}\right\} .
\end{aligned}
$$

Comme dans la référence [23], nous introduisons les coefficients $d^{(k)}$ définis par :

$$
\left\{\begin{array}{l}
{ }^{J} T_{0}^{(0)}=d^{(0)} \\
{ }_{J} T_{0}^{(1)}=d^{(1)} J_{z} \\
{ }^{J} T_{0}^{(2)}=\frac{1}{\sqrt{6}} d^{(2)}\left[3 J_{z}^{2}-J(J+1)\right] .
\end{array}\right.
$$

Les coefficients $d^{(k)}$ dépendent de $J$ et $J_{1}$ et sont calculés dans le paragraphe suivant.

Un calcul semblable à celui de la référence [23] nous permet alors de calculer $L\left(\sigma_{+}\right)$et $L\left(\sigma_{-}\right)$(intensité de la lumière émise par les atomes dans la direction de $\mathbf{B}_{0}$ avec une polarisation circulaire droite ou gauche), ainsi que $L(\pi)$ et $L(\sigma)$ (intensité émise perpendiculairement à $\mathbf{B}_{0}$ avec une polarisation linéaire soit parallèle, soit perpendiculaire à $\mathbf{B}_{0}$ ) :

$$
\left\{\begin{array}{l}
L\left(\sigma_{+}\right)=\alpha \Gamma\left\langle-\frac{d^{(2)}}{6}\left[3 J_{z}^{2}-J(J+1)\right]-\right. \\
\left.-\frac{d^{(0)}}{\sqrt{3}}-\frac{d^{(1)}}{\sqrt{2}} J_{z}\right\rangle_{s} \\
L\left(\sigma_{-}\right)=\alpha \Gamma\left\langle-\frac{d^{(2)}}{6}\left[3 J_{z}^{2}-J(J+1)\right]-\right. \\
\left.-\frac{d^{(0)}}{\sqrt{3}}+\frac{d^{(1)}}{\sqrt{2}} J_{z}\right\rangle s \quad(\mathrm{I} . \mathrm{B} .22) \\
L(\pi)=\alpha \Gamma\left\langle-\frac{d^{(0)}}{\sqrt{3}}+\frac{d^{(2)}}{3}\left[3 J_{z}^{2}-J(J+1)\right]\right\rangle_{s} \\
L(\sigma)=\alpha \Gamma\left\langle-\frac{d^{(0)}}{\sqrt{3}}-\frac{d^{(2)}}{6}\left[3 J_{z}^{2}-J(J+1)\right]\right\rangle_{s}
\end{array} .\right.
$$

Pour obtenir cette expression de $L(\sigma)$ nous avons utilisé le fait que, le système étant invariant par rotation autour de $O z$, l'égalité $\left\langle J_{x}^{2}\right\rangle_{s}=\left\langle J_{y}^{2}\right\rangle_{s}$ entraîne

$\left.\left.<3 J_{x}^{2}-J(J+1)\right\rangle_{s}=-\frac{1}{2}<3 J_{z}^{2}-J(J+1)\right\rangle_{s}$.

(10) Le changement de signe dans (I.B.20) du coefficient de $\left\langle{ }^{J} T_{0}^{(1)}\right\rangle$ par rapport à l'équation (III.13) de [22] provient du fait que $\mathbf{e}_{\lambda}$ correspond, dans l'expression de $L\left(\mathbf{e}_{\lambda}\right)$ (intensité lumineuse émise par les atomes), à $\mathbf{e}_{\lambda_{0}}^{*}$ dans celle de $L_{A}\left(\mathbf{e}_{\lambda_{0}}\right)$ (intensité lumineuse absorbée par les atomes).
Les taux de polarisation $\mathfrak{T}\left(\sigma_{+}, \sigma_{-}\right)$et $\mathfrak{T}(\pi, \sigma)$ sont définis par

$$
\left\{\begin{array}{l}
\mathfrak{T}\left(\sigma_{+}, \sigma_{-}\right)=\frac{L\left(\sigma_{+}\right)-L\left(\sigma_{-}\right)}{L\left(\sigma_{+}\right)+L\left(\sigma_{-}\right)} \\
\mathfrak{T}(\pi, \sigma)=\frac{L(\pi)-L(\sigma)}{L(\pi)+L(\sigma)} .
\end{array}\right.
$$

On remarque sur (I.B.22) que les sommes des intensités lumineuses, qui figurent aux dénominateurs des quantités $\mathfrak{T}$, dépendant non seulement de la population totale du niveau excité (terme en $d^{(0)}$ ) mais encore de l'alignement

$$
\text { (terme en } \left.d^{(2)}<3 J_{z}^{2}-J(J+1)>_{s}\right) \text {. }
$$

Dans les expériences réalisées en pratique, l'orientation $P$ de l'état fondamental est faible : il en est donc de même de l'alignement $<3 J_{z}^{2}-J(J+1)>_{s}$, dont nous allons négliger la contributon aux dénominateurs des taux de polarisation $\mathfrak{T}$, devant celle de la population totale. (Il n'est pas difficile de constater expérimentalement que l'approximation ainsi faite est bonne : il suffit de vérifier que la quantité totale de lumière mesurée dans une direction donnée par un détecteur non sensible à la polarisation varie très peu lorsque l'on crée la polarisation nucléaire dans l'état fondamental). Nous obtenons alors, en remarquant que $\left\langle d^{(0)}\right\rangle_{s}=\operatorname{Tr}\left\{d^{(0)} \rho^{s}\right\}=d^{(0)} / \Gamma T_{\mathrm{d}}$ (cf. équation (I. A. 4)) :

$$
\left\{\begin{aligned}
\mathfrak{T}\left(\sigma_{+}, \sigma_{-}\right) & \left.=\sqrt{\frac{3}{2}} \frac{d^{(1)}}{d^{(0)}} \Gamma T_{\mathrm{d}}<J_{z}\right\rangle_{s} \\
\mathfrak{T}(\pi, \sigma) & \left.=-\frac{\sqrt{3}}{4} \frac{d^{(2)}}{d^{(0)}} \Gamma T_{\mathrm{d}}<3 J_{z}^{2}-J(J+1)\right\rangle_{s}
\end{aligned}\right.
$$

b) Calcul des coefficients $d^{(k)}$. - Il reste pour terminer le calcul, à déterminer les coefficients $d^{(0)}, d^{(1)}$ et $d^{(2)}$. Dans ce but, nous allons transformer la relation (I. B. 19) en y reportant la relation de définition de $\mathbf{D}$ (cf. références [22] et [23]) :

$$
\left\{\begin{array}{cc}
<J & m_{J}\left|D_{q}\right| J_{1} m_{J}^{\prime}>=\left\langle J m_{J}\right| J_{1} 1 m_{J}^{\prime} q> \\
\left\langle J_{1} m_{J}\left|D_{q}\right| J m_{J}^{\prime}>=\right. & \text { (I. B. } \\
=(-1)^{q}<J m_{J} \mid J_{1} 1 m_{J}^{\prime}-q>
\end{array}\right.
$$

Nous obtenons alors :

$$
\begin{aligned}
<J m_{J}\left|{ }^{J} T_{q}^{(k)}\right| J m_{J}^{\prime}>=\sum_{\substack{q^{\prime}+q^{\prime \prime}=q \\
m_{J}^{\prime \prime}}}<11 q^{\prime} q^{\prime \prime} \mid k q>\times \\
\quad \times<J m_{J} \mid J_{1} 1 m_{J}^{\prime \prime} q^{\prime}> \\
\quad \times(-1)^{q^{\prime \prime}}<J_{1} 1 m_{J}^{\prime \prime}-q^{\prime \prime} \mid J m_{J}^{\prime}>
\end{aligned}
$$

soit, tous calculs faits :

$$
\begin{aligned}
< & \left.J m_{J}\right|^{J} T_{q}^{(k)} \mid J m_{J}^{\prime}>=\sqrt{(2 k+1)(2 J+1)} \times \\
& \times(-1)^{k+J+J_{1}}\left\{\begin{array}{lll}
J & J & k \\
1 & 1 & J_{1}
\end{array}\right\}<J k m_{J}^{\prime} q \mid J m_{J}>.
\end{aligned}
$$


- Calcul de $d^{(0)}$.

Lorsque $k=0$, le coefficient $6 j$ de la relation (I. B. 27) vaut

$$
(-1)^{1+J+J_{1}} \frac{1}{\sqrt{3(2 J+1)}}
$$

et on a donc :

$$
d^{(0)}=-1 / \sqrt{3} .
$$

- Calcul de $d^{(1)}$.

Calculons par exemple :

$$
\begin{gathered}
<m_{J}\left|{ }^{J} T_{0}^{(1)}\right| m_{J}>=\sqrt{3(2 J+1)}(-1)^{1+J+J_{1}} \times \\
\times\left\{\begin{array}{lll}
J & J & 1 \\
1 & 1 & J_{1}
\end{array}\right\}<J 1 m_{J} 0 \mid J m_{J}>.
\end{gathered}
$$

Le coefficient de Clebsch-Gordan de cette expression vaut :

$$
\begin{aligned}
<J 1 m_{J} 0 \mid J m_{J}> & =\frac{m_{J}}{\sqrt{J(J+1)}} \\
& =\frac{1}{\sqrt{J(J+1)}}<m_{J}\left|J_{z}\right| m_{J}>
\end{aligned}
$$

et on a donc :

$$
d^{(1)}=(-1)^{1+J+J_{1}} \sqrt{\frac{3(2 J+1)}{J(J+1)}}\left\{\begin{array}{lll}
J & J & 1 \\
1 & 1 & J_{1}
\end{array}\right\} .
$$

- Calcul de $d^{(2)}$.

Un calcul analogue fournit la valeur de $d^{(2)}$ :

$$
\begin{aligned}
d^{(2)}=(-1)^{J+J_{1}} & \sqrt{\frac{30(2 J+1)}{(2 J-1) J(J+1)(2 J+3)}} \times \\
& \times\left\{\begin{array}{lll}
J & J & 2 \\
1 & 1 & J_{1}
\end{array}\right\} .
\end{aligned}
$$

Il suffit maintenant de regrouper les diverses formules obtenues plus haut pour obtenir les expressions définitives des taux de polarisation lumineuse $\left({ }^{11}\right)$

$$
\left\{\begin{array}{l}
\mathfrak{T}\left(\sigma_{+}, \sigma_{-}\right)=(-1)^{J+J_{1}} \sqrt{\frac{27}{2}} \sqrt{\frac{2 J+1}{J(J+1)}} \times \\
\quad \times\left\{\begin{array}{lll}
J & J & 1 \\
1 & 1 & J_{1}
\end{array}\right\} \frac{P}{(4 J+2)} \sum_{m_{F}} f_{m_{F}}\left(B_{0}\right) \\
\quad \times \sqrt{\frac{(\mathrm{I}}{(2}(\pi, \sigma)}=(-1)^{J+J_{1}} \sqrt{\frac{135}{8}} \times \\
\times\left\{\begin{array}{lll}
J & J & 2 \\
1 & 1 & J_{1}
\end{array}\right\} \frac{6 P}{(4 J+2)} \sum_{m_{F}} m_{F} f_{m_{F}}\left(B_{0}\right) .
\end{array}\right.
$$

Rappelons que la définition de $f_{m_{F}}\left(B_{0}\right)$ est donnée en (I.B.14).

(11) La formule (I.B.30) appliquée dans le cas où $a \gg \Gamma$, $B_{0}=0, J=1$ et $J_{0}=0$, donne $\mathfrak{T}\left(\sigma_{+}, \sigma_{-}\right)=\frac{8}{18} P$.

Nous retrouvons le résultat du paragraphe I.A.2.c.
C. Application, dans le cas $\mathbf{J}=\mathbf{2}$, à la mesure de la structure hyperfine. - Dans ce paragraphe les résultats, obtenus plus haut dans le cas général, sont explicités lorsque les nombres quantiques $J$ et $J_{1}$ valent respectivement 2 et 1 ; c'est en effet le cas que nous avons étudié expérimentalement (transition $n{ }^{1} D_{2}$ $2{ }^{1} P_{1}$ de ${ }^{3} \mathrm{He}$ ). Lorsque $J=2$, il existe quatre valeurs de $B_{0}$ qui correspondent à un anticroisement dans le niveau excité : les courbes, qui donnent les variations en fonction de $B_{0}$ de $\mathfrak{T}\left(\sigma_{+}, \sigma_{-}\right)$et $\mathfrak{T}(\pi, \sigma)$, sont donc obtenues par superposition de quatre courbes de Lorentz, de largeurs différentes, mais symétriques deux à deux par rapport à la valeur $B_{0}=0$. La figure 5 montre les courbes ainsi obtenues, tracées dans le cas $\gamma=0$; le maximum de $\mathfrak{T}\left(\sigma_{+}, \sigma_{-}\right)$, obtenu pour la valeur $B_{0}=0$, a pour valeur

$$
\mathcal{T}_{\max }\left(\sigma_{+}, \sigma_{-}\right)=0,24 P
$$

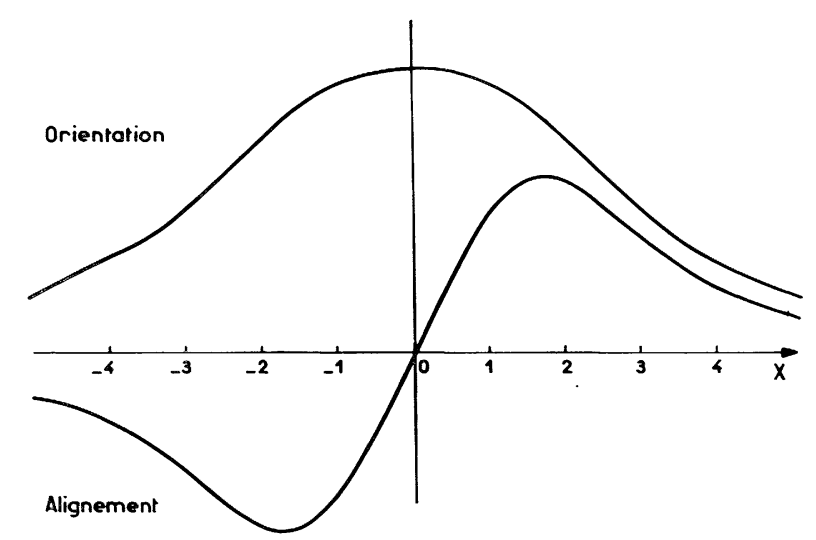

FIG. 5. - Variations théoriques en fonction de $B_{0}$ de l'orientation $\left\langle J_{z}\right\rangle_{s}$ et de l'alignement $\left\langle J(J+1) / 3-J_{z}^{2}\right\rangle_{s}$ dans un niveau de nombre quantique $J=2$. Le paramètre $x$ sans dimension est défini par $x=g_{J} \mu_{B} B_{0} /\left[\left(J+\frac{1}{2}\right) \hbar \Delta W\right]\left(g_{J}\right.$, facteur de Landé ; $\mu_{B}$, magnéton de Bohr ; $\hbar \Delta W$, structure hyperfine) ; lorsque $x$ est de l'ordre de 1 , le découplage hyperfin est partiel.

On a supposé $\Delta W . \tau \gg 1$ ( $\tau$, durée de vie), c'est-à-dire $\gamma \simeq 0$. Ces courbes sont la superposition de plusieurs courbes de Lorentz, avec des poids positifs ou négatifs.

alors que le maximum de $\mathfrak{T}(\pi, \sigma)$, obtenu en champ $B_{0}$ intermédiaire $x \simeq(1,73)$, vaut :

$$
\mathcal{T}_{\max }(\pi, \sigma) \simeq 0,089 P .
$$

Nous verrons plus bas qu'il est commode, pour des raisons expérimentales, de mesurer les variations en fonction de $B_{0}$ du rapport $R=\mathfrak{T}(\pi, \sigma) / \mathfrak{T}\left(\sigma_{+}, \sigma_{-}\right)$. Les courbes théoriques correspondantes sont données sur la figure 6, pour différentes valeurs de $\gamma$. Supposons que l'on puisse obtenir expérimentalement une telle courbe; on peut penser à en tirer une mesure de $\gamma$ et $a$, c'est-à-dire de la durée de vie et de la structure hyperfine du niveau. Remarquons toutefois que les variations des courbes théoriques en fonction de $\gamma$ sont du second ordre; pour les petites valeurs de $\gamma$ (ce qui est le cas pour les niveaux atomiques que nous avons étudiés), la détermination expérimentale de la 
durée de vie par cette méthode serait très imprécise. Il vaut donc mieux chercher à éliminer $\gamma$, et ne mesurer que la structure hyperfine, grâce à la relation $x=\alpha B_{0} / a$ où $x$ est connu par comparaison avec les courbes théoriques, $\alpha$ est connu par le calcul du facteur de Landé du niveau, $B_{0}$ est connu expérimentalement.

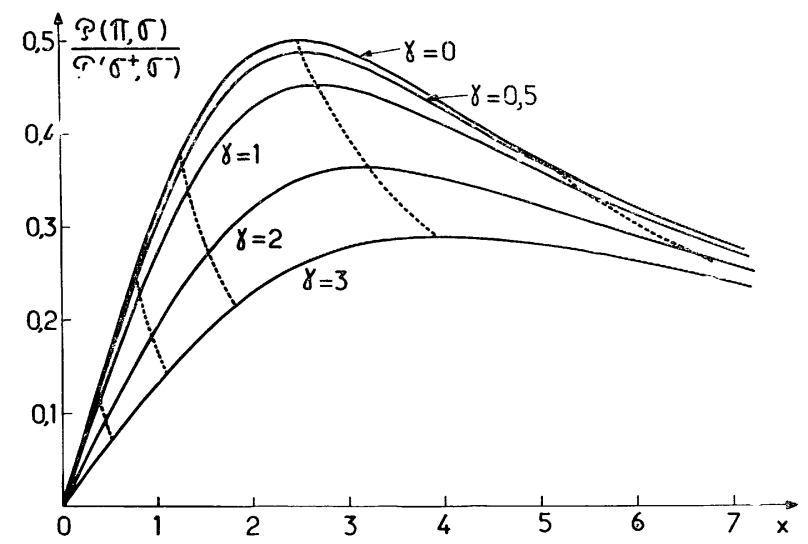

FIG. 6. - Variations théoriques en fonction de $B_{0}$ du rapport $R$ entre $\mathfrak{T}(\pi, \sigma)$, taux de polarisation linéaire, et $\mathfrak{T}\left(\sigma_{+}, \sigma_{-}\right)$, taux de polarisation circulaire. $x$ est défini dans la légende de la figure précédente; le paramètre $\gamma$ est défini par : $\gamma=\Gamma / a=(a \tau)^{-1}$. Lorsque $\gamma=0$, la valeur maximum $R_{m}$ de $R$ est $R_{m}=0,5$, obtenue pour $x \simeq 0,776$. On voit sur cette figure que lorsque $\gamma$ est petit devant $1, R$ dépend peu de $\gamma$ (les variations de $R$ sont du second ordre en $\gamma$ ). Les courbes en pointillés joignent les points où $R$ prend sa valeur maximum, les $3 / 4$, la moitié, ou le quart de cette valeur. On remarque que l'abscisse $x^{*}$, pour laquelle $R$ prend la moitié de sa valeur maximum, dépend peu de $\gamma$ lorsque $\gamma$ est petit.

Il reste à déterminer la valeur de $x$ qui donne les meilleurs résultats; on peut éliminer la valeur $x_{m}$ qui rend $R$ maximum $\left(R=R_{m}\right)$ car, si elle permet de bien connaître $R_{m}$, elle ne donne la valeur correspondante de $B_{0}$ qu'avec une grande incertitude. Nous avons préféré choisir la valeur $x^{*}$ pour laquelle $R$ vaut $\left(\frac{1}{2}\right) R_{m}$. Cette valeur de $x$ présente deux avantages :

- $R$ varie rapidement lorsque $x \simeq x^{*}$, de sorte que la valeur expérimentale $B_{0}^{*}$ de $B_{0}$ correspondante peut être déterminée expérimentalement avec une bonne précision.

- $x^{*}$ dépend peu de $\gamma$ de sorte que la mesure de la structure hyperfine ainsi obtenue dépendra peu de la durée de vie du niveau (tant que $\gamma$ reste inférieur à 1) ; la figure 7 montre les variations de $x^{*}$ en fonction de $\gamma$. On voit que, si l'on peut par exemple affirmer que $\gamma$ a une valeur inférieure à 0,5 , on commet une erreur de l'ordre de $1 \%$ en supposant que $\gamma$ est nul (ce qui revient à prendre $x^{*}=0,776$ ).

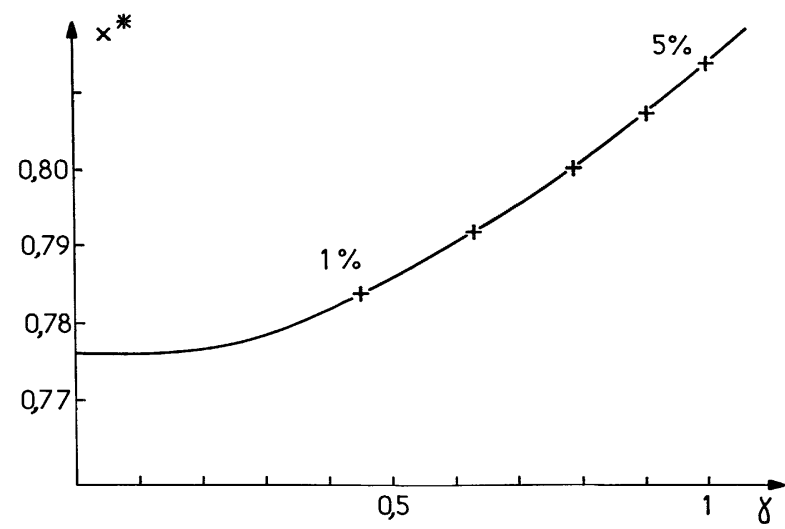

Fig. 7. - Variations de $x^{*}$ en fonction de $\gamma$. Tant que $\gamma$ est inférieur à 0,5 on fait une erreur de l'ordre de $1 \%$ en prenant pour $x^{*}$ la valeur 0,776 correspondant à $\gamma=0$; lorsque $\gamma$ vaut 1,1 'erreur ainsi faite reste faible, de l'ordre de $5 \%$.

\section{II. ÉTUDE EXPÉRIMENTALE}

La méthode étudiée dans cet article est très générale et peut être appliquée à de nombreux éléments possédant un isotope de spin nucléaire non nul. Nous avons vu plus haut que le choix du procédé d'excitation des atomes (décharge dans un gaz) nous a conduits à appliquer la méthode dans le cas de ${ }^{3} \mathrm{He}$. Cette partie est consacrée à la description des expériences qui ont été réalisées.

A. Description des expériences. - Le pompage optique de ${ }^{3} \mathrm{He}$ présente quelques particularités. Aussi consacrons nous un premier paragraphe à quelques rappels.

1. Pompage optique de ${ }^{3} \mathrm{He}$ - - La méthode d'orientation par pompage optique du spin nucléaire de ${ }^{3} \mathrm{He}$ a été mise au point par Colegrove, Schearer et Walters [13]. Ces auteurs n'ont pas utilisé la raie de résonance de l'hélium, car elle ne convient pas au pompage optique pour deux raisons :

- cette raie est située dans l'ultraviolet relativement lointain $(\lambda=585 \AA)$, et il est difficile expérimentalement d'obtenir un faisceau lumineux de cette longueur d'onde polarisé circulairement ;

- la structure hyperfine du niveau $2{ }^{1} P$ de ${ }^{3} \mathrm{He}$ (niveau de résonance) est extrêmement faible, de même que sa durée de vie [24], de sorte que l'on se trouve dans le cas où la condition $a \tau \ll 1$ est réalisée ; l'orientation par pompage optique du spin nucléaire est alors impossible [5].

Aussi Colegrove et al. ont-ils procédé différemment : ayant peuplé, au moyen d'une faible décharge entretenue dans le gaz d'hélium, le niveau métastable triplet $2{ }^{3} S_{1}$ (cf. Fig. 8), ils ont orienté ce niveau par pompage optique grâce à la raie spectrale de longueur 

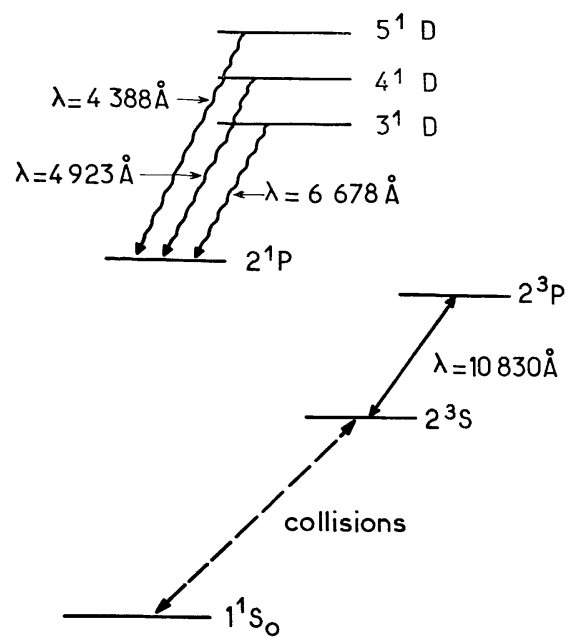

FIG. 8. - Niveaux de ${ }^{3} \mathrm{He}$ intervenant dans cette expérience. Le pompage de ${ }^{3} \mathrm{He}$ est assuré par la raie spectrale $\lambda=10830 \AA$. La détection de l'orientation nucléaire de l'état fondamental est réalisée par la mesure du taux de polarisation de la raie $\lambda=6678 \AA$, ou des autres raies issues des autres niveaux ${ }^{1} D$.

d'onde $\lambda=10830 \AA$. On évite ainsi les deux difficultés évoquées plus haut :

- la raie $\lambda=10830 \AA$ tombe dans l'infrarouge proche ; elle peut aisément être polarisée (filtres polarisants "Polaroïd» du type H. R.) et les lampes à hélium l'émettent avec une forte intensité ;

- le niveau métastable que l'on oriente par pompage optique possède un moment angulaire électronique $J=1$, et une structure hyperfine importante de plusieurs milliers de mégahertz; le spin nucléaire peut donc être orienté au cours du cycle de pompage optique, par exemple au cours de l'étape "évolution propre dans le niveau métastable ».

L'orientation ainsi obtenue dans le niveau métastable $2{ }^{3} S_{1}$ est ensuite transférée au niveau fondamental $1{ }^{1} S_{0}$, lors de collisions dites « d'échange de métastabilité»: au cours d'une telle collision entre un atome dans l'état $2{ }^{3} S_{1}$, orienté par pompage optique, et un atome non orienté dans l'état $1{ }^{1} S_{0}$, l'orientation peut être transférée du niveau métastable au niveau fondamental. Le couplage ainsi obtenu entre les orientations de ces deux niveaux est très fort ; toute variation de la polarisation de l'un d'entre eux entraîne rapidement une variation de la polarisation dans l'autre. C'est ainsi que ce couplage permet, non seulement d'orienter le niveau $1{ }^{1} S_{0}$ à partir du niveau $2{ }^{3} S_{1}$, mais encore de détecter par l'intermédiaire du niveau $2{ }^{3} S_{1}$ l'orientation du niveau $1{ }^{1} S_{0}$; il suffit pour cela d'utiliser un faisceau lumineux polarisé circulairement de longueur d'onde $\lambda=10830 \AA$, et de mesurer les variations relatives $\Delta L_{A} / L_{A}$ de la lumière de ce faisceau absorbée par les atomes dans l'état métastable. Le faisceau lumineux détecté doit être issu d'une lampe remplie de l'isotope ${ }^{4} \mathrm{He}$; les coincidences isotopiques de la raie $\lambda=10830 \AA$ sont telles que, dans ce cas, seules certaines composantes de structure fine ou hyperfine de la transition $2{ }^{3} S \rightarrow 2{ }^{3} P$ sont utilisées pour la détection optique (on peut montrer que ces diverses composantes ne donnent pas toutes des signaux de même signe, et que le signal que l'on obtiendrait en supposant non résolues l'ensemble de ces composantes serait nul $\left({ }^{12}\right)$. Notons toutefois que cette méthode de détection est peu précise; en effet les déplacements isotopiques de la raie $\lambda=10830 \AA$ sont relativement mal connus; de plus, les structures hyperfines correspondantes sont de l'ordre de quelques largeurs Doppler, de sorte qu'il est difficile de savoir exactement quelles transitions de ${ }^{3} \mathrm{He}$ peuvent être induites par une lampe à ${ }^{4} \mathrm{He}$, sans connaître exactement la forme de la raie spectrale émise par cette lampe. Pour ces raisons, la relation entre $\Delta L_{A} / L_{A}$ et la polarisation nucléaire $P$ de l'état fondamental est sujette à discussions [13], [25], [26].

Rappelons enfin une dernière particularité du pompage optique de ${ }^{3} \mathrm{He}$ : la pression à l'intérieur de la cellule qui contient l'hélium est beaucoup plus forte que celles que l'on utilise habituellement dans le pompage optique d'autres éléments (mercure, alcalins, etc...) ; la raison de cette différence est la suivante: dans une décharge faible, il y a environ $10^{5}$ ou $10^{6}$ fois moins d'atomes d'hélium dans le niveau métastable que dans l'état fondamental ; le nombre d'atomes soumis directement à l'action du faisceau de pompage est donc du même ordre de grandeur que dans les expériences sur d'autres éléments. La forte valeur de la pression d'hélium a une conséquence sur les expériences que nous avons réalisées : la désorientation par collisions dans les divers niveaux excités n'est pas toujours négligeable.

2. DesCription du montage. - Le schéma général du montage est donné sur la figure 9. Nous n'en donnons ici qu'une description sommaire en nous limitant à quelques points essentiels; on pourrait trouver plus de détails dans la référence [16].

a) La cellule. - La cellule est une sphère de pyrex, de diamètre $6 \mathrm{~cm}$, qui contient le gaz de ${ }^{3} \mathrm{He}$ très pur, sous une pression comprise entre $1 / 10$ de torr et quelques torrs.

b) La décharge. - Le rôle de cette décharge est de peupler le niveau métastable $2{ }^{3} S_{1}$, et d'exciter par collisions les atomes dans de nombreux niveaux, d'où ils retombent en émettant diverses raies spectrales. Il est commode d'utiliser une décharge H. F., afin d'éviter l'emploi d'électrodes internes à la cellule. La fréquence d'excitation ne joue à priori aucun rôle particulier. Nous avons toutefois été conduits à faire un grand nombre d'essais avec des procédés d'excitation et des fréquences différentes, avant de réussir à satis-

(12) C'est pour une raison analogue que, lorsque la pression d'hélium dans la cellule dépasse quelques dixièmes de torr, et que le niveau $2{ }^{3} P$ est fortement désorienté par collisions (pompage optique du type ((Dehmelt) ) [1], [27]), il faut utiliser une lampe à ${ }^{4} \mathrm{He}$ pour réaliser le pompage optique de ${ }^{3} \mathrm{He}$. 


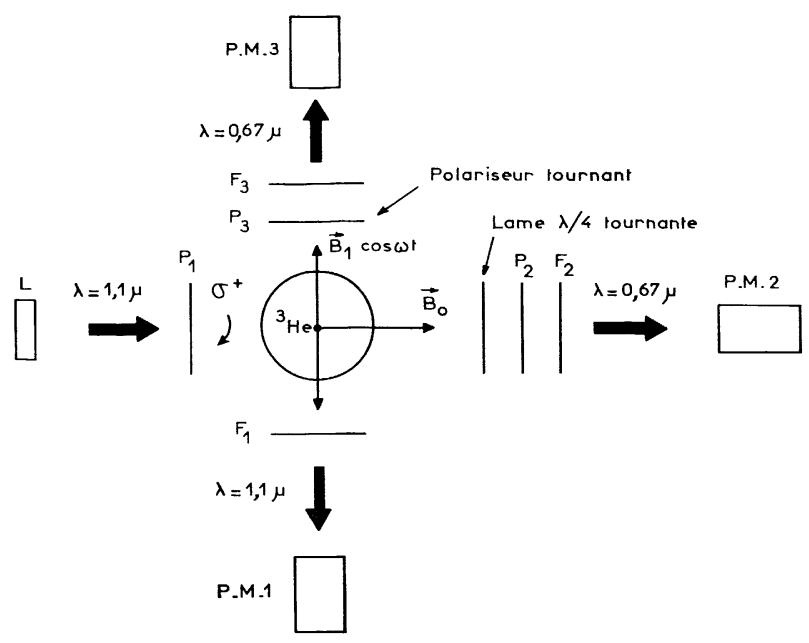

FIG. 9. - Schéma général du montage, montrant le faisceau de pompage de longueur d'onde $\lambda=1,1 \mu$, le faisceau de détection de même longueur d'onde, et deux faisceaux de détection servant à mesurer les taux de polarisation $\mathfrak{T}\left(\sigma_{+}, \sigma_{-}\right)$et $\mathfrak{T}(\pi, \sigma)$ des différentes raies spectrales émises par la décharge (ici $\lambda=0,67 \mu)$.

$L \quad$ : Lampe à ${ }^{3} \mathrm{He}$ ou ${ }^{4} \mathrm{He}$, suivant que la pression dans la cellule est inférieure ou supérieure à 0,3 torr.

$P_{1} \quad$ : Polariseur circulaire (polaroïd H. R.), doublé par un filtre Wratten $87 \mathrm{C}$ ne laissant passer que l'infrarouge.

$\mathrm{F}_{1} \quad$ : Filtre Kodak Wratten $87 \mathrm{C}$.

$F_{2}, F_{3}$ : Filtres interférentiels permettant d'isoler une des raies spectrales émises par la décharge (ici, $\lambda=0,67 \mu$ ).

$\mathrm{P}_{2} \quad$ : Polariseur fixe.

$\mathbf{P}_{3} \quad$ : Polariseur tournant.

$B_{0} \quad$ : Champ magnétique statique; $B_{1} \cos \omega t$ est un champ de radio fréquence permettant de détruire l'orientation nucléaire de l'état fondamental.

PM1 : Photomultiplicateur de photocathode du type S1.

PM2 et PM3 : photomultiplicateurs de photocathodes S20.

faire les trois conditions suivantes : la décharge doit être très stable; elle doit être isotrope (en l'absence de pompage optique, la lumière qu'elle émet ne doit pas être polarisée) ; enfin, et c'est la condition la plus difficile à obtenir, elle doit ne pas être affectée par un champ magnétique statique dont la valeur peut être comprise entre 0 et 200 gauss. Les essais nous ont conduits à adopter la solution suivante, pour laquelle les diverses conditions requises sont réalisées de manière acceptable : un générateur de quelques watts et de fréquence $14 \mathrm{MHz}$ débite dans l'enroulement primaire d'un transformateur élévateur de tension; on utilise ensuite un fil conducteur dont une extrémité est connectée à un pôle du secondaire, et dont l'autre arrive à quelques millimètres de la cellule. Le circuit secondaire est accordé grâce à une capacité variable.

c) Les lampes. - La partie émissive est constituée par un disque de pyrex de $30 \mathrm{~mm}$ de diamètre; ce disque est relié par un capillaire à un réservoir d'hélium, afin d'éviter une disparition trop rapide de gaz par diffusion à travers les parois. La pression d'hélium sous laquelle ces lampes sont remplies est de l'ordre de 6 torrs; suivant les cas on utilise l'isotope ${ }^{3} \mathrm{He}$ ou ${ }^{4} \mathrm{He}$ (cf. note 12 ).

d) Les champs magnétiques. - Une paire de bobines en position d'Helmholtz permet de créer un champ magnétique statique $\mathbf{B}_{0}$ dont l'intensité peut varier entre 0 et 400 gauss. Une paire de bobines auxiliaires est utilisée pour obtenir un champ oscillant $\mathbf{B}_{1} \cos \omega t$, perpendiculaire à $\mathbf{B}_{0}$, ce qui permet d'effectuer une résonance magnétique sur le niveau fondamental ${ }^{1} S_{0}$.

e) Ladétection optique. - Raie spectrale $\lambda=10830 \AA$. Nous avons vu qu'une détection lumineuse à cette longueur d'onde permet de mesurer, de façon indirecte, la polarisation nucléaire $P$ de l'état fondamental. La mesure des variations de $L_{A}$, intensité du faisceau de pompage absorbée par les atomes, présente divers inconvénients (fond continu important dû aux composantes émises par une lampe à ${ }^{4} \mathrm{He}$ non absorbables par la vapeur de ${ }^{3} \mathrm{He}$; impossibilité de placer un autre faisceau détecteur dans la direction de $\mathbf{B}_{0}$ ).

Afin d'obtenir une meilleure précision, nous avons préféré mesurer les variations relatives $\Delta L_{F} / L_{F}$ de l'intensité de la lumière du faisceau de pompage diffusée par les atomes dans un angle solide donné (lumière de fluorescence). Cet angle solide étant inférieur à $4 \pi$, on a : $L_{F}<L_{A}$; toutefois, on peut montrer par le calcul que $\Delta L_{F} / L_{F}=\Delta L_{A} / L_{A}\left({ }^{13}\right)$, et les signaux donnés par des mesures d'absorption ou de fluorescence sont théoriquement équivalents. Expérimentalement on évite ainsi les inconvénients qui viennent d'être signalés, ce qui compense largement le fait que $L_{F}$ soit plus petit que $L_{A}$. Le faisceau lumineux correspondant est représenté sur la figure 9 , ainsi que le photomultiplicateur PM1 qui mesure $L_{F}$. Malgré les précautions prises, cette méthode reste peu précise pour mesurer $P$, pour diverses raisons. La première est l'incertitude, signalée plus haut sur la relation entre $P$ et $\Delta L_{A} / L_{A}$; les autres raisons sont d'ordre expérimental : les photomultiplicateurs sont très peu sensibles dans le domaine spectral de l'infra-rouge ; de plus les signaux obtenus sont continus, et l'on est gêné par de nombreux effets parasites (cf. Fig. 12) (dérives, effets de fatigue du photomultiplicateur, etc...) enfin, la mesure de $L_{F}$ est obtenue par différence de deux intensités lumineuses plus grandes que $L_{F}$ (signaux obtenus en éteignant la décharge soit en présence du faisceau de pompage, soit en son absence) et, là encore les effets de fatigue rendent cette mesure imprécise.

- Autres raies spectrales émises par la décharge.

Deux faisceaux lumineux, représentés sur la figure 9 (dans le cas particulier où $\lambda=0,67 \mu$ ), sont utilisés afin de mesurer les taux de polarisation $\mathcal{T}\left(\sigma_{+}, \sigma_{-}\right)$et $\Im(\pi, \sigma)$. Nous avons étudié successivement un certain

(13) Cette relation se démontre en utilisant le fait qu'un faisceau lumineux issu d'une lampe à ${ }^{4} \mathrm{He}$ ne peut faire subir aux atomes de ${ }^{3} \mathrm{He}$ que des transitions entre le niveau métastable $2{ }^{3} S_{1}$ et le sous-niveau hyperfin $F=\frac{1}{2}$ du niveau $2{ }^{3} P_{0}$, les autres niveaux $2{ }^{3} P$ étant exclus [13], [16], [28]; la lampe à ${ }^{4} \mathrm{He}$ ne crée donc aucun alignement dans le niveau excité $2{ }^{3} P$. 
nombre de raies émises par la décharge; un filtre interférentiel convenable (noté $\mathrm{F}_{2}$ ou $\mathrm{F}_{3}$ sur la Fig. 9) permet d'isoler chacune d'entre elles. Une des difficultés de l'expérience consiste à mesurer de faibles taux de polarisation $\mathcal{T}$. Dans ce but, nous avons utilisé l'appareillage suivant (cf. Fig. 10) : la lumière émise par la

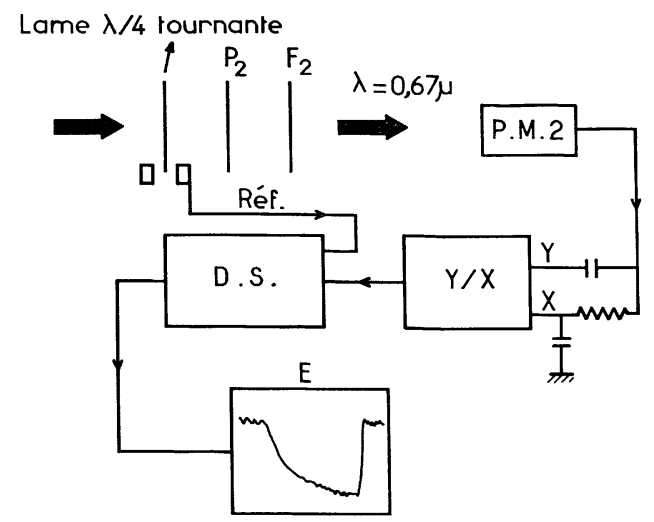

Fig. 10. - Schéma du montage permettant de mesurer, avec un bon rapport signal sur bruit, le taux de polarisation $\int\left(\sigma_{+}, \sigma_{-}\right)$. La lame $\lambda / 4$ tournante constitue avec le polariseur $P_{2}$ fixe un analyseur qui est alternativement droit et gauche. Le filtre interférentiel $F_{2}$ permet d'isoler la raie spectrale choisie. Le photomultiplicateur PM2 détecte une modulation lumineuse superposée à un fond continu; le ratiomètre $Y / X$ effectue le rapport entre ces deux quantités. La détection synchrone D. S., dont la référence est commandée par la rotation de la lame $\lambda / 4$, permet d'améliorer le rapport signal sur bruit. Le signal obtenu est envoyé sur un enregistreur $\mathrm{E}$.

décharge traverse une lame biréfringente $\lambda / 4$ tournante, effectuant $N$ tours par seconde, dans son plan, autour de l'axe du faisceau lumineux; cette lumière traverse ensuite un polariseur $\mathrm{P}_{2}$ fixe, le filtre $\mathrm{F}_{2}$, et tombe sur le photomultiplicateur PM2. L'ensemble constitué par la lame $\lambda / 4$ tournante et le polariseur fixe correspond successivement à un analyseur circulaire droit, gauche, et ainsi de suite. On comprend que, si la lumière incidente possède un certain taux de polarisation circulaire $\mathfrak{T}\left(\sigma_{+}, \sigma_{-}\right)$, le photomultiplicateur PM2 détecte une modulation lumineuse de fréquence $2 N$, proportionnelle à $\mathfrak{S}\left(\sigma_{+}, \sigma_{-}\right)$et à l'intensité lumineuse totale émise par la décharge $\left({ }^{14}\right)$. Le signal électrique délivré par PM2 est ensuite dirigé sur un « ratiomètre », qui effectue le rapport entre la partie modulée de ce signal et sa partie continue : on obtient alors une tension alternative de fréquence $2 N$ qui n'est plus proportionnelle qu'à $\mathfrak{T}\left(\sigma_{+}, \sigma_{-}\right)$; cette tension est ensuite envoyée dans une détection synchrone dont la référence est commandée par un signal qui a exactement la fréquence $2 N$ (ce signal est issu d'un dispositif électrique et optique convenable adjoint à la lame $\lambda / 4$ tournante). Le montage ainsi réalisé permet d'obtenir directement le taux de polarisation $\mathfrak{T}\left(\sigma_{+}, \sigma_{-}\right)$, et d'éliminer les fluctuations de l'intensité totale émise par la décharge,

(14) Le calcul exact de l'amplitude de cette modulation et de sa phase est donné en appendice. ainsi que les dérives du photomultiplicateur. La sensibilité de ce dispositif permet de détecter de faible taux de polarisation lumineuse, de l'ordre de $10^{-4}$ lorsque la décharge n'est pas trop faible et que la raie étudiée tombe dans un domaine spectral où les photomultiplicateurs ont une bonne sensibilité.

Le montage qui vient d'être décrit peut facilement être adapté à la détection des taux de polarisation $\mathcal{T}(\pi, \sigma)$; il suffit de remplacer la lame $\lambda / 4$ par un polariseur tournant et de supprimer le polariseur fixe.

Précisons enfin que la suspension de la lame $\lambda / 4$ tournante et sa rotation sont assurées par un dispositif utilisant un " coussin d'air »; seul ce montage nous a permis d'obtenir une rotation suffisamment rapide $(2 N$ peut varier de 60 à $250 \mathrm{~Hz}$ ) et stable, d'un polariseur de grand diamètre $(12 \mathrm{~cm}$ utiles), sans faire appel à aucun matériau magnétique.

3. Mise en Évidence des Signaux. - Nous avons utilisé le montage décrit dans le paragraphe précédent pour étudier la polarisation d'un certain nombre des raies lumineuses émises par une décharge dans ${ }^{3} \mathrm{He}$. Certaines de ces expériences ont déjà fait l'objet de Comptes Rendus [29]. Pour mettre en évidence le phénomène recherché, nous avons choisi la raie de longueur d'onde $\lambda=6678 \AA$, issue du niveau $3{ }^{1} \mathrm{D}$; la structure hyperfine de ce niveau $\Delta W / 2 \pi \simeq 138 \mathrm{MHz}$ a été mesurée par Descoubes et al. [12], [30] ; sa durée de vie a été calculée ou mesurée par divers auteurs (références [30] à [33]) et est de l'ordre de $1,5 \times 10^{-8} \mathrm{~s}$; le produit $\Delta W . \tau$ est donc de l'ordre de 10 . Il s'ensuit que la raie $\lambda=6678 \AA$ correspond à un cas où la polarisation nucléaire $P$ de l'état fondamental doit effectivement se traduire par une polarisation lumineuse $\mathfrak{T}\left(\sigma_{+}, \sigma_{-}\right)$.

La première expérience consiste simplement à enregistrer les variations de $\mathfrak{S}\left(\sigma_{+}, \sigma_{-}\right)$en fonction $\mathrm{du}$ temps, au moment où l'on commence à effectuer le pompage optique de ${ }^{3} \mathrm{He}$; la figure 11 montre l'enregistrement ainsi obtenu ; à la fin de l'enregistrement, on a appliqué un champ de radiofréquence intense à la fréquence de résonance de l'état fondamental, de façon à détruire brusquement la polarisation nucléaire $P$. On constate effectivement que les variations de $\mathfrak{T}\left(\sigma_{+}, \sigma_{-}\right)$reflètent bien à chaque instant les variations de la polarisation nucléaire $P$.

Nous avons vu que, pour obtenir un taux de polarisation linéaire $\mathfrak{T}(\pi, \sigma)$, il est nécessaire de créer un découplage hyperfin partiel dans le niveau $3{ }^{1} D$, au moyen d'un champ magnétique statique $\mathbf{B}_{0}$ (cf. $\S$ I.A. $2 b$ ) ; la valeur de la structure hyperfine de ce niveau montre que $B_{0}$ doit être de l'ordre de 100 gauss. Nous avons vérifié que, dans un tel champ, une polarisation linéaire $\mathfrak{T}(\pi, \sigma)$ apparaît effectivement, alors qu'elle n'existe pas en champ magnétique nul ou très intense (cas du découplage total de $\mathbf{I}$ et $\mathbf{J}$ ). Enfin nous avons vérifié que, lorsque l'on inverse le sens de $\mathbf{B}_{0}, \Im(\pi, \sigma)$ change de signe (cf. Fig. 5).

Nous avons ensuite cherché à montrer que cette nou- 


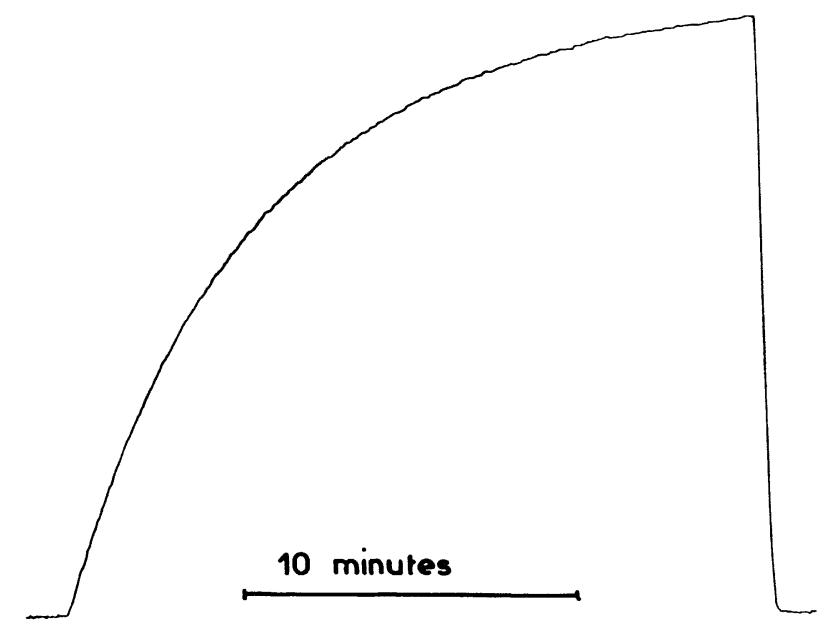

FIG. 11. - Variation du taux de polarisation $\mathfrak{T}\left(\sigma_{+}, \sigma_{-}\right)$(raie spectrale $\lambda=6678 \AA$ ) en fonction du temps, au moment où l'on commence le pompage optique dans l'état fondamental. La constante de temps de pompage, très longue, est caractéristique du pompage optique de ${ }^{3} \mathrm{He}$, effectué par l'intermédiaire du niveau métastable $2{ }^{3} S_{1}$. La polarisation nucléaire limite obtenue est de l'ordre de $12 \%$, correspondant à une polarisation lumineuse $\mathfrak{T}\left(\sigma_{+}, \sigma_{-}\right) \simeq 2 \%$. A la fin de l'enregistrement, on a appliqué un champ de radiofréquence de façon à annuler brusquement la polarisation nucléaire $P$. Les conditions de l'expérience sont les suivantes : champ magnétique $B_{0} \simeq 10$ gauss, pression dans la cellule $p=0,8$ torr, constante de temps de la détection synchrone $3 \mathrm{~s}$ donnant un rapport signal sur bruit de l'ordre de 100 ; la décharge dans la cellule est très faible.

velle méthode permet d'orienter plusieurs niveaux excités de ${ }^{3} \mathrm{He}$. Dans ce but, nous avons étudié un certain nombre de raies spectrales émises par la décharge. Nous avons constaté l'existence d'une polarisation lumineuse $\mathfrak{T}\left(\sigma_{+}, \sigma_{-}\right)$des raies $\lambda=6678,4923$ et $4388 \AA$, mettant en évidence une orientation électronique dans les niveaux $3{ }^{1} \mathrm{D}, 4^{1} \mathrm{D}$ et $5^{1} \mathrm{D}$. La même mesure portant sur les raies $\lambda=5876$ et $4471 \AA$ nous a montré l'existence de cette orientation dans les niveaux triplets correspondants $3{ }^{3} D$ et $4{ }^{3} D$. Enfin, une étude de la raie $\lambda=3888 \AA$ nous a donné le même résultat positif concernant le niveau $3{ }^{3} P$. Par contre, nous avons obtenu un résultat négatif dans les cas suivants : $\lambda=7281 \AA$ (niveau $3{ }^{1} S$ ), $7065 \AA$ (niveau $3{ }^{3} S$ ), $4713 \AA$ (niveau $4{ }^{3} S$ ) et $5016 \AA$ (niveau $3{ }^{1} P$ ). Dans le cas des niveaux ${ }^{1} S$, on comprend aisément que le moment électronique $\mathbf{J}$ est nul et ne peut être orienté. Dans le cas des niveaux ${ }^{3} S$, on peut remarquer que, lorsque le moment orbital $\mathbf{L}$ est nul, la méthode que nous employons ne peut orienter que le spin électronique; or les divers effets d'une telle orientation sur la polarisation de l'ensemble des composantes de structure fine de la raie lumineuse s'annulent entre eux, de sorte que la lumière de fluorescence globale n'est pas polarisée. Dans le cas du niveau ${ }^{1} P$, on peut penser que la très courte durée de vie de ce niveau de résonance $\left(\tau \simeq 1,8 \times 10^{-9} \mathrm{~s}[34]\right)$ et sa très faible structure hyperfine, de l'ordre du mégahertz [35], qui donnent un produit $\Delta W \times \tau<10^{-2}$, sont suffisamment faibles pour expliquer le résultat négatif obtenu.
La méthode que nous utilisons peut ainsi être considérée comme un moyen sensible de détecter une orientation nucléaire dans l'état fondamental. La comparaison des signaux obtenus de cette manière avec ceux que donne la méthode classique utilisant la longueur d'onde $\lambda=10830 \AA$ (cf. Fig. 12) montre l'amélioration des signaux que permet ce procédé de détection. Un autre avantage de ce procédé réside dans le fait que $\mathfrak{T}\left(\sigma_{+}, \sigma_{-}\right)$est directement proportionnel à $P$, alors que la relation entre $\Delta L_{A} / L_{A}$ et $P$ est plus compliquée.

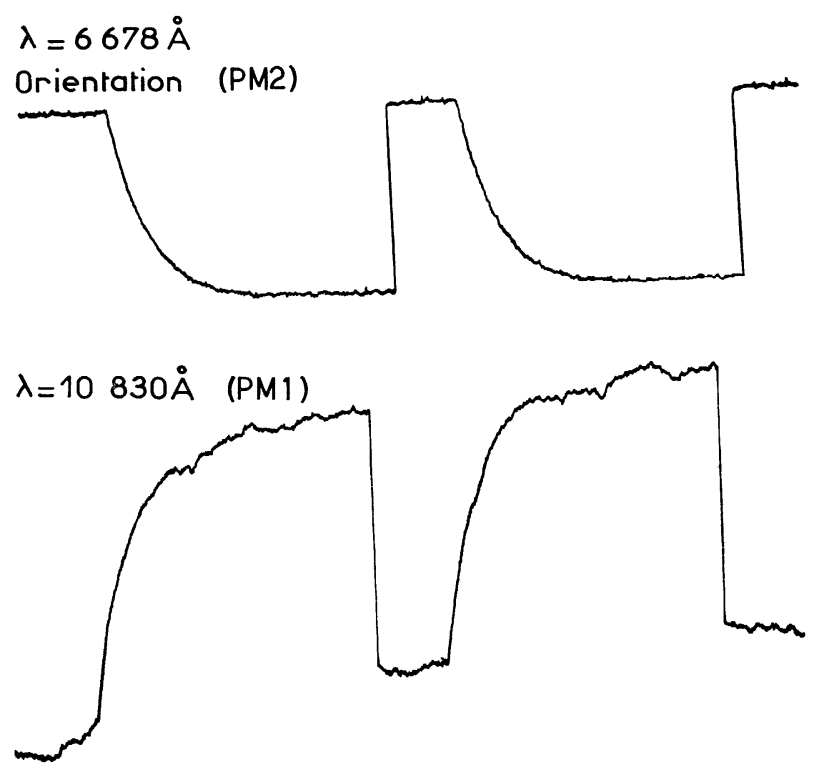

FIG. 12. - Enregistrement simultané des signaux donnés par PM1 et PM2 ; au début de l'enregistrement, on a commencé le pompage optique; une fois l'orientation obtenue, on a appliqué un champ de radiofréquence pour détruire la polarisation nucléaire $P$; ensuite, on a répété la même séquence. La durée totale de l'enregistrement est de 8 minutes. Condition de l'expérience : $B_{0}=10$ gauss; pression dans la cellule : 0,5 torr ; constante de temps de la détection synchrone du faisceau $2: 300 \mathrm{~ms}$. On voit que, même avec cette faible constante de temps, les signaux obtenus avec une détection sur la raie $\lambda=6678 \AA$ sont bien meilleurs que ceux de la raie $\lambda=10830 \AA$.

4. ETUde DE $\mathcal{T}\left(\sigma_{+}, \sigma_{-}\right)$ET $\mathcal{T}(\pi, \sigma)$ EN FONCTION DE $B_{0}$. - Il est nécessaire, lorsque l'on désire étudier expérimentalement les variations des taux de polarisation lumineuse $\mathfrak{T}\left(\sigma_{+}, \sigma_{-}\right)$et $\mathfrak{T}(\pi, \sigma)$ en fonction du champ magnétique, de mesurer pour chaque valeur de $B_{0}$ la polarisation nucléaire $P$. En effet, cette polarisation peut varier en fonction de $B_{0}$, notamment à cause des variations très importantes du temps de relaxation nucléaire dans l'état fondamental, dues à l'influence des inhomogénéités magnétiques [13], [37] [38]. C'est la raison pour laquelle nous avons simultanément mesuré dans nos expériences les signaux $\mathfrak{T}\left(\sigma_{+}, \sigma_{-}\right), \mathfrak{T}(\pi, \sigma)$ et $\Delta L_{F} / L_{F}$. La mesure des taux de polarisation $\mathcal{T}$ demande certaines précautions, qui permettent d'éliminer les polarisations lumineuses parasites (anisotropies de la décharge, réflexion des faisceaux lumineux, etc...) ; il suffit de mesurer les varia- 
tions de la polarisation de la lumière détectée par les photomultiplicateurs, lorsque l'on détruit brusquement la polarisation nucléaire de l'état fondamental par un champ de radiofréquence (cf. Fig. 12 et 13). La mesure de $\Delta L_{F} / L_{F}$ nous a permis de calculer $P$ par la

$$
\lambda=6678 \AA
$$

Orientation (PM2)

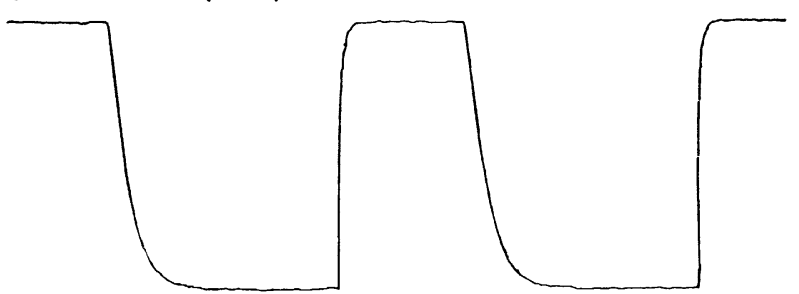

Alignement (PM3)

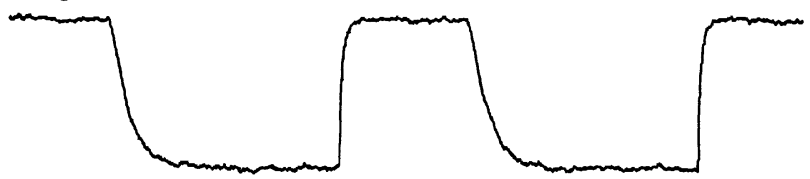

FIG. 13. - Enregistrement simultané des variations de $\mathfrak{T}\left(\sigma_{+}, \sigma_{-}\right)$ et $\mathfrak{T}(\pi, \sigma)$ en fonction du temps. Conditions de l'expérience : $B_{0}=20$ gauss ; pression dans la cellule 0,095 torr ; raie spectrale étudiée $\lambda_{0}=6678 \AA$. Pour les raies spectrales $\lambda=4923 \AA$ et $\lambda=4388 \AA$, nous avons obtenu un rapport signal sur bruit tout à fait comparable.

relation approchée $P \simeq \frac{2}{5} \Delta L_{F} / L_{F}$, valable lorsque $P$ est faible [13] [25] [26] ; il est alors possible de porter, en fonction de $B_{0}$, les valeurs des rapports $\mathfrak{T} / P$. Les figures 14 et 15 montrent les résultats expérimentaux ainsi obtenus ; la comparaison avec la figure 5 montre que l'accord entre la théorie et l'expérience est assez bon. On voit sur ces figures que, pour une polarisation nucléaire $P$ de l'ordre de $10 \%$, on obtient :

$$
\begin{aligned}
& \mathfrak{T}_{\max }\left(\sigma_{+}, \sigma_{-}\right) \simeq 2 \% \\
& \mathfrak{T}_{\text {max }}(\pi, \sigma) \simeq 0,6 \% .
\end{aligned}
$$

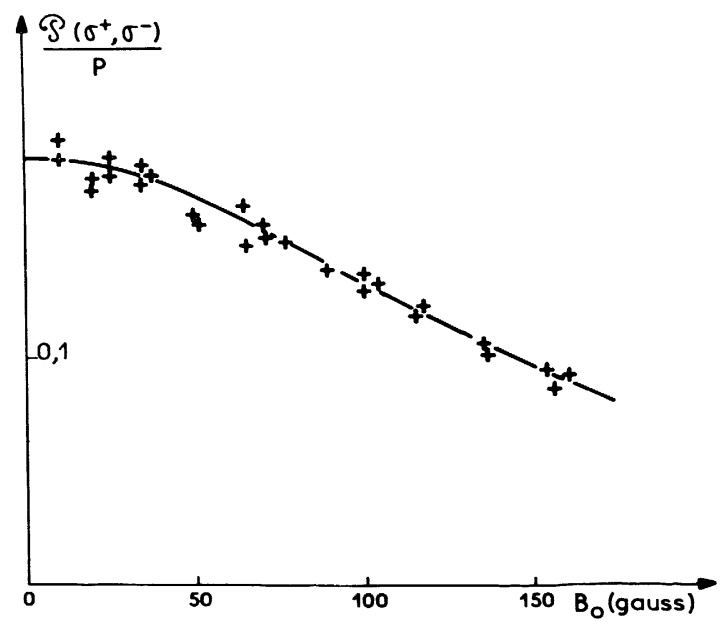

Fig. 14. - Variation, en fonction du champ magnétique statique, du rapport $\mathcal{S}\left(\sigma_{+}, \sigma_{-}\right) / P$. La dispersion des points expérimentaux provient de l'imprécision de la mesure de $P$.

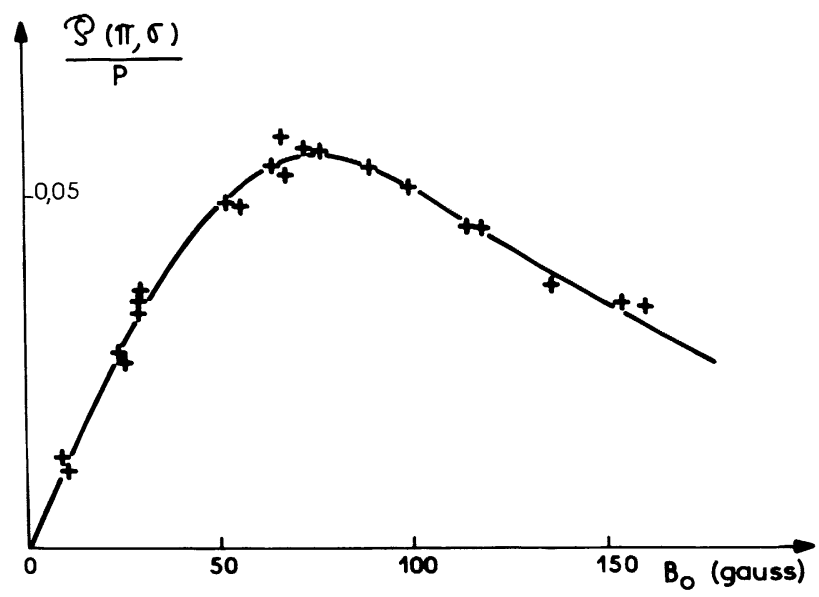

Fig. 15. - Variation, en fonction du champ magnétique statique, du rapport $\mathfrak{S}(\pi, \sigma) / P$.

Là encore, l'accord avec les prévisions théoriques (cf. équation (I. C. 1) et (I. C. 2)) est acceptable, compte tenu de l'imprécision que comporte une mesure absolue de taux de polarisation $\mathcal{T}$, effectuée avec des polariseurs et lames $\lambda / 4$ imparfaites.

On peut penser à ajuster les échelles horizontales des figures 14 (ou 15) et 5 pour les faire coïncider : on pourrait ainsi connaître la structure hyperfine $\Delta W$ du niveau étudié ; on constate toutefois que la dispersion des résultats ainsi obtenus est grande (de l'ordre de $\pm 20 \%$ ). Nous attribuons cette imprécision à la mauvaise connaissance de $P$. On peut alors tenter d'améliorer ces résultats en étudiant expérimentalement les variations du rapport $R=\mathfrak{T}(\pi, \sigma) / \mathfrak{T}\left(\sigma_{+}, \sigma_{-}\right)$, ce qui permet d'éliminer $P$. Les expériences correspondantes sont décrites dans le paragraphe suivant.

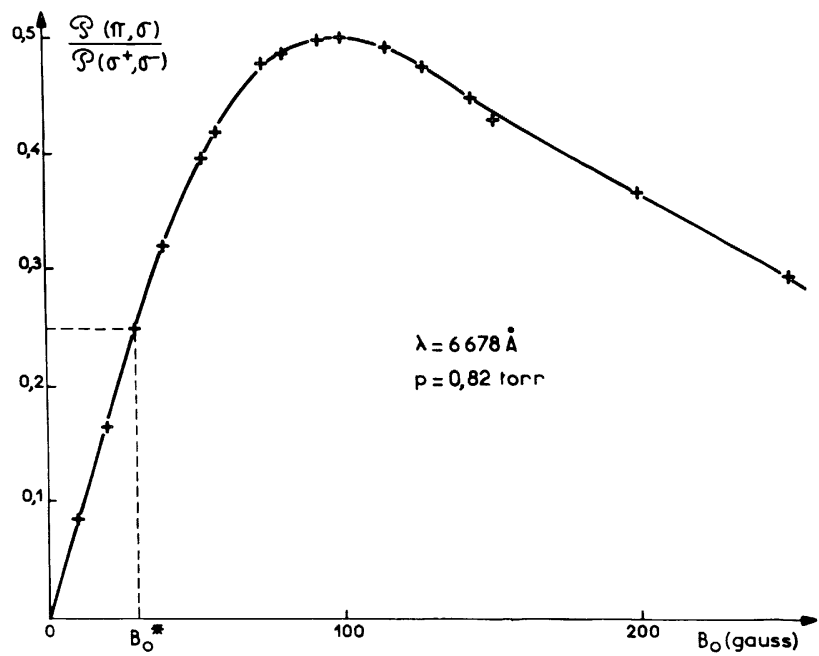

Fig. 16. - Mesure en fonction du champ magnétique $B_{0}$ du rapport entre les taux de polarisation $\mathfrak{T}(\pi, \sigma)$ et $\mathfrak{T}\left(\sigma_{+}, \sigma_{-}\right)$. Les points sont expérimentaux, la courbe théorique. L'ajustement de l'échelle horizontale a permis de calculer la structure hyperfine du niveau $3^{1} D_{2}$ (le résultat trouvé dans cette série de mesures est $138 \mathrm{MHz}$ ). On voit sur cette figure que l'élimination de $\mathbf{P}$ permet d'obtenir une dispersion des points expérimentaux beaucoup plus faible que sur les figures 14 et 15 . 
B. Mesure de structure hyperfine. - 1. PRINCIPE DE LA MÉTHODE. - Le principe de la méthode de mesure des structures hyperfines consiste à étudier expérimentalement les variations, en fonction du champ magnétique $B_{0}$, du rapport $R=\mathfrak{T}(\pi, \sigma) / \mathfrak{T}\left(\sigma_{+}, \sigma_{-}\right)$. On peut ainsi obtenir la valeur $B_{0}^{*}$ de $B_{0}$ pour laquelle $R$ prend une valeur égale à la moitié de son maximum $R_{\mathrm{m}}$; on sait que la valeur $B_{0}=B_{0}^{*}$ correspond à $x=x^{*}=0,776$ (cf. § I. C) ; la structure hyperfine, exprimée en mégahertz, est donc donnée par :

$$
10^{-6} \cdot \frac{\Delta W}{2 \pi}=\frac{10^{-6}\left(J+\frac{1}{2}\right)}{0,776} \cdot \frac{g_{J} \mu_{B} B_{0}^{*}}{\hbar} .
$$

La figure 16 montre les résultats expérimentaux d'une étude de $R$ en fonction de $B_{0}$, pour la raie spectrale $\lambda=6678 \AA$. La valeur de $B_{0}^{*}$ ainsi obtenue a permis de calculer la structure hyperfine du niveau $3{ }^{1} D_{2}$ de ${ }^{3} \mathrm{He}$, ce qui donne $138 \mathrm{MHz}$. La courbe en trait plein a été calculée à partir de cette valeur, c'est-àdire en faisant coïncider les points théorique et expérimental $B_{0}=B_{0}^{*}$ et $R=R_{\mathrm{m}} / 2\left({ }^{15}\right)$. On voit que, grâce à l'élimination de $P$, la dispersion des points est beaucoup moins grande et que la coïncidence entre les points et la courbe pleine est bonne.

Nous avons effectué des vérifications analogues avec des pressions diverses d'hélium et sur les raies $\lambda=6678 \AA, \lambda=4923 \AA$ et $\lambda=4388 \AA$; nous avons ainsi vérifié avec une bonne précision l'accord entre la théorie et l'expérience. Signalons toutefois que, avec certaines intensités de la décharge, et dans des champs $B_{0}$ supérieurs à 150 gauss, nous avons pu mettre en évidence un léger désaccord, de l'ordre de $5 \%$, avec les courbes théoriques; nous attribuons ce désaccord à l'action directe du champ magnétique sur la décharge qu'il rend anisotrope ; nous pensons qu'il n'affecte que très peu les mesures de structures hyperfines, qui ne dépendent que des points pour lesquels $B_{0} \leqslant 100$ gauss.

2. Structure hyperfine des NiveauX ${ }^{1} D$ DE ${ }^{3} \mathrm{He}$. Dans ce paragraphe, nous rappelons l'origine de la structure hyperfine des niveaux ${ }^{1} D$ de ${ }^{3} \mathrm{He}$, ainsi que les résultats des mesures antérieures de ces structures. Les configurations électroniques des états excités de l'atome d'hélium sont du type $(1 s, n l)$, de sorte que l'atome possède toujours un électron interne dont la probabilité de présence sur le noyau est grande ; il s'ensuit que pour cet électron, le terme de contact $2 a \mathbf{I} . \mathbf{S}_{1}$ de l'hamiltonien hyperfin est très important, et que l'on peut négliger la contribution des autres

(15) La figure 16 montre que les variations relatives de $R$ correspondent bien aux précisions théoriques. On peut ainsi chercher à effectuer la même vérification sur les valeurs absolues de $R$. On constate alors que la valeur théorique de $R_{\mathrm{m}}$ est, pour un niveau $J=2$, égale à 0,5 (dans le cas où $\gamma \ll 1$ ); expérimentalement, on obtient 0,42 . Cette valeur légèrement plus faible peut s'expliquer par les imperfections des lames $\lambda / 4$ et des polariseurs, déjà évoquées plus haut ; on peut aussi faire intervenir les difficultés d'étalonnage des deux voies électroniques de mesure (détections synchrones, etc...), qui peuvent perturber une mesure absolue des taux de polarisation $\mathfrak{T}$. termes. Dans un niveau de nombre quantique $S$ donné, le théorème de Wigner-Eckart permet alors d'écrire : $\mathbf{S}_{1}=\frac{1}{2} \mathbf{S}$ et $\mathbf{H}_{\mathrm{hyp}}=a \mathbf{I} . \mathbf{S}$. Cet opérateur, agissant dans les états triplets de ${ }^{3} \mathrm{He}$, permet de calculer leur structure hyperfine, qui est très importante ; par contre, cet opérateur donne 0 dans les états singulets. Aussi a-t-on pensé un certain temps que la structure hyperfine des singulets de ${ }^{3} \mathrm{He}$ était très faible. C'est alors que des expériences de Decomps et al. [39] [40] ont montré que ces structures n'étaient pas si faibles; ces auteurs ont même donné une évaluation de leur valeur pour quelques niveaux $n^{1} D$, comprise suivant les cas entre 50 et $100 \mathrm{MHz}$.

L'origine de cette structure est la suivante : sous l'influence de l'hamiltonien hyperfin, il se produit un certain « mélange » entre les états ${ }^{1} D$ et ${ }^{3} D$, de sorte que $S$ n'est plus un bon nombre quantique; ce « mélange » permet de faire apparaître dans les niveaux singulets une partie de la structure hyperfine des triplets, et ceci avec d'autant plus d'efficacité que le triplet et le singulet sont proches. Le calcul théorique correspondant a été fait par Moser et al. [41] ainsi que par Descoubes [12], et donne des structures comprises entre 50 et $150 \mathrm{MHz}$.

L'association des techniques de «bombardement électronique » et de " croisements de niveaux » a permis à Descoubes [12] de mesurer des structures hyperfines de niveaux ${ }^{1} D$; certains des résultats correspondants sont portés sur le tableau I. On voit que la précision de ces mesures est bonne; signalons d'ailleurs que, devant la difficulté que présente parfois l'identification des divers " croisements », cet auteur avait été conduit à donner trois valeurs possibles de la structure hyperfine de chaque niveau ${ }^{1} D$; toutefois des considérations théoriques, ainsi que des mesures antérieures de la durée de vie de ces niveaux [39] [40] lui avaient permis d'adopter l'une de ces trois valeurs (marquée d'un astérisque sur le tableau). Nous verrons plus bas que nos résultats permettent de confirmer que, dans tous les cas, son choix était le bon $\left({ }^{16}\right)$.

3. RÉSUltats OBTENUS - Discussion. - Les résultats que nous avons obtenus sont portés sur le tableau I. On voit que l'accord avec les valeurs données par Descoubes est bon. On peut se demander pourquoi la précision de nos mesures n'est pas supérieure à celle des méthodes de "croisements de niveaux », alors que le rapport signal sur bruit obtenu est meilleur (cf. Fig. 13). La raison de cette imprécision provient de ce que notre méthode fait intervenir des « anticroisements » de niveaux, dont la largeur est beaucoup plus grande que celle des « croisements».

On peut discuter la méthode de mesure qui a été exposée en faisant les remarques suivantes : le calcul théorique sur lequel cette méthode s'appuie suppose

(16) Signalons que des nouvelles expériences de croisement de niveaux, effectuées récemment par Descoubes et Dily, ont permis d'identifier sans ambiguïté tous les croisements de niveaux, et de confirmer ces valeurs des structures hyperfines. 
que les diverses collisions (atomiques, électroniques, ioniques) que subit un atome pendant son passage dans l'état excité affectent peu l'orientation et l'alignement de cet état. De plus, le calcul suppose que, lorsqu'un atome parvient dans l'état excité, il ne possède en moyenne qu'une orientation nucléaire (et non électronique); cela suppose notamment que la décharge est isotrope (hypothèse déjà discutée plus haut $\left({ }^{17}\right)$, et qu'il n'existe pas d'effet de cascade à partir d'un autre niveau où le couplage hyperfin soit assez grand pour orienter $\mathbf{J}\left({ }^{18}\right)$. Pour appuyer ces deux hypothèses, nous avons effectué des mesures de structures hyperfines en faisant varier indépendamment la pression de ${ }^{3} \mathrm{He}$ contenue dans la cellule (de 0,1 à 1 torr) et le niveau de décharge dans le gaz (l'intensité des raies émises variant dans un facteur supérieur à 5). Les valeurs obtenues tombent toutes dans les marges d'erreur indiquées sur le tableau ; on peut donc penser que les hypothèses faites sont bien réalisées.

Conclusion. - La méthode exposée dans cet article constitue un moyen très général d'étude des niveaux atomiques excités; en effet, l'excitation par décharge constitue un procédé commode qui permet d'atteindre aisément un très grand nombre de niveaux. Les expériences qui ont été exposées peuvent être prolongées dans plusieurs directions : on peut chercher à

(17) Nous avons supposé dans le calcul théorique que la décharge est isotrope, afin de pouvoir écrire (cf. équations I. A.6 et I.A.7) :

$$
\begin{array}{lll}
\rho^{0}=\rho_{n}^{0} \otimes \rho_{e}^{0} \text { avec } \quad \begin{array}{l}
\rho_{n}^{0}=\frac{1}{2}+P I_{z} ; \\
\rho_{e}^{0}=1 / 2(J+1)
\end{array}
\end{array}
$$

Il est possible de s'affranchir de cette hypothèse, et de supposer que la décharge n'est pas isotrope et crée dans le niveau excité un certain alignement macroscopique; il faut alors écrire, si l'on suppose par exemple que cet alignement est longitudinal :

$$
\rho_{e}^{0}=\frac{1}{2 J+1}\left[1+Q_{J}^{0} T_{0}^{(2)}\right]
$$

$\rho^{s}$, qui est fonction linéaire de $\rho^{0}$ (cf. I. A, 4).comprend alors quatre termes :

un terme qui ne dépend ni de $P$ ni de $Q$,

un terme proportionnel à $P$,

un terme proportionnel à $Q$,

un terme proportionnel à $P Q$.

Les variations des signaux optiques lorsque l'on annule $P$ ne dépendent que du deuxième et du quatrième terme; les effets du deuxième terme sont ceux qui sont étudiés dans cet article ; quant au quatrième terme, il est négligeable, car $P$ et $Q$ sont des quantités petites : en effet $P$ est de l'ordre de 5 à $10 \%$; l'alignement $Q$ créé par la décharge peut être évalué en mesurant le taux de polarisation de la lumière émise par la décharge, en l'absence de pompage optique; nous avons vérifié expérimentalement que cette polarisation lumineuse ne dépasse en aucun cas 1 ou $2 \%$.

(18) Dans le cas des niveaux $1 D$, une orientation électronique ne pourrait provenir directement par cascade que des niveaux $\boldsymbol{1} \boldsymbol{P}$ ou ${ }^{1} F$. Notons toutefois que l'effet des niveaux ${ }^{1} P$, dont la structure hyperfine est très petite, est certainement négligeable [rappelons que l'orientation et l'alignement électroniques sont, lorsque la condition $a \tau \ll 1$ est réalisée, proportionnels à $(a \tau)^{2}$ ].
TABLEAU I

\begin{tabular}{|c|c|}
\hline${ }^{1} D \ldots 146 \pm 3$ & $\begin{array}{c}86,8 \pm 1,5 \\
109 \pm 2 \\
* 138 \pm 2,5\end{array}$ \\
\hline${ }^{1} D \ldots 110 \pm 3$ & $\begin{array}{r}64,3 \pm 0,6 \\
80,8 \pm 0,8 \\
* 102 \pm 1\end{array}$ \\
\hline${ }^{1} D \ldots 103$ & $\begin{array}{r}59,0 \pm 0,6 \\
74,2 \pm 0,7 \\
* 93,8 \pm 0,9\end{array}$ \\
\hline
\end{tabular}

Structures hyperfines (en mégahertz)

$\begin{array}{ccc}\begin{array}{c}\text { Calculées } \\ \text { par Moser } \\ {[41]}\end{array} & \begin{array}{c}\text { Mesurées par } \\ \text { Descoubes } \\ {[12],[30]}\end{array} & \begin{array}{c}\text { Mesures } \\ \text { exposées } \\ \text { dans cet } \\ \text { article }\end{array}\end{array}$

réaliser des mesures par résonance magnétique dans les états excités et à effectuer ainsi des mesures de durées de vie, de sections efficaces de collision, etc... dans un domaine de pressions qui complètera les résultats antérieurs [12], [33], [42] à [45]. On peut aussi tenter d'orienter par pompage optique les spins nucléaires d'autres gaz rares et appliquer ensuite la même méthode. On peut enfin chercher à l'étendre à d'autres éléments comme par exemple le mercure ou le cadmium. De telles expériences sont en cours au laboratoire.

Remerciements. - Les auteurs remercient le Professeur Kastler de les avoir accueillis dans son laboratoire, les Professeurs Brossel et Cohen-Tannoudji d'avoir bien voulu diriger de très près ce travail. Ils remercient aussi tous les ingénieurs et techniciens du laboratoire dont la collaboration a été précieuse pour réaliser ces expériences et notamment : Madame Berland et Monsieur Legaut qui ont conçu et réalisé divers dispositifs électroniques, notamment les « ratiomètres »; Madame Dousset et Monsieur Camy qui ont participé à la mise au point de l'appareillage permettant de réaliser des décharges très stables et indépendantes du champ magnétique; Messieurs Cassou et Guillaume qui ont réalisé les dispositifs mécaniques permettant la rotation des lames quart d'onde sur coussin d'air.

Appendice. - Calcul des signaux donnés par la lame quart d'onde tournante.

Nous considérons le montage suivant (cf. Fig. 10) : une lame biréfringente, de retard $\varphi$, tourne autour de $O Z$ avec la vitesse angulaire $\omega(O Z$ est la direction de propagation du faisceau lumineux, $O X Y Z$ un trièdre trirectangle direct); un polariseur fixe transmet les polarisations linéaires parallèles à un axe $O X$.

On appelle $\left|\mathbf{e}_{X}\right\rangle$ et $\left|\mathbf{e}_{Y}\right\rangle$ les vecteurs polarisation correspondant aux polarisations linéaires parallèles à $O X$ et $O Y$, et on pose :

$$
\left\{\begin{array}{l}
\left|\mathbf{e}_{u}\right\rangle=\frac{1}{\sqrt{2}}\left[\left|\mathbf{e}_{X}\right\rangle+\left|\mathbf{e}_{Y}\right\rangle\right] \\
\left|\mathbf{e}_{v}\right\rangle=\frac{1}{\sqrt{2}}\left[\left|\mathbf{e}_{X}\right\rangle=\left|\mathbf{e}_{Y}\right\rangle\right]
\end{array}\right.
$$


$\left|\mathbf{e}_{u}\right\rangle$ et $\left|\mathbf{e}_{v}\right\rangle$ correspondent aux polarisations linéaires à 45 degrés de $O X$ et $O Y$.

$$
\left\{\begin{array}{l}
\left|\mathbf{e}_{+}\right\rangle=-\frac{1}{\sqrt{2}}\left[\left|\mathbf{e}_{X}\right\rangle+i\left|\mathbf{e}_{Y}\right\rangle\right] \\
\left|\mathbf{e}_{-}\right\rangle=\frac{1}{\sqrt{2}}\left[\left|\mathbf{e}_{X}\right\rangle-i\left|\mathbf{e}_{Y}\right\rangle\right]
\end{array}\right.
$$

$\mid \mathbf{e}^{+}>$et $\left|\mathbf{e}^{-}\right\rangle$correspondent aux polarisations circulaires droite et gauche.

La polarisation lumineuse incidente est définie par la matrice polarisation $\pi$, de vecteurs propres orthogonaux $\left|\mathbf{e}_{\lambda}\right\rangle$ et $\left|\mathbf{e}_{\lambda}^{\prime}\right\rangle ; \pi$ s'écrit :

$\pi=I_{\lambda}\left|\mathbf{e}_{\lambda}><\mathbf{e}_{\lambda}\right|+I_{\lambda^{\prime}}\left|\mathbf{e}_{\lambda^{\prime}}><\mathbf{e}_{\lambda^{\prime}}\right|\left(I_{\lambda} \geqslant I_{\lambda^{\prime}}\right)$

ou encore :

$$
\pi=I_{\lambda^{\prime}}+\left(I_{\lambda}-I_{\lambda^{\prime}}\right)\left|\mathbf{e}_{\lambda}><\mathbf{e}_{\lambda}\right| .
$$

Le premier terme correspond à la partie non polarisée de $\pi$, le second à la partie polarisée : le taux de polarisation est $\left(I_{\lambda}-I_{\lambda}^{\prime}\right) /\left(I_{\lambda}^{\prime}+I_{\lambda}^{\prime}\right)$.

On peut caractériser le vecteur $\left|\mathbf{e}_{\lambda}\right\rangle$ par les paramètres $\theta$ et $\psi$ en posant :

$$
\left|\mathbf{e}_{\lambda}\right\rangle=\mathrm{e}^{-i \theta} \cos \psi\left|\mathbf{e}_{X}\right\rangle+\mathrm{e}^{i \theta} \sin \psi\left|\mathbf{e}_{Y}\right\rangle .
$$

Soit $M_{\varphi}(t)$ la matrice associée à la lame biréfringente ; après traversée de cette lame, la matrice polarisation est:

avec :

$$
\pi^{\prime}=M_{\varphi}(t) \pi M_{\varphi}^{+}(t)
$$

$$
M_{\varphi}(t)=\left|\mathbf{e}_{1}(t)><\mathbf{e}_{1}(t)\right|+e^{i \varphi}\left|\mathbf{e}_{2}(t)><\mathbf{e}_{2}(t)\right|
$$

où $\left|\mathbf{e}_{1}(t)\right\rangle$ et $\left|\mathbf{e}_{2}(t)\right\rangle$ définissent respectivement les axes rapide et lent de la lame, et sont donnés par :

$$
\left\{\begin{array}{l}
\left|\mathbf{e}_{1}(t)\right\rangle=\cos \omega t\left|\mathbf{e}_{X}\right\rangle+\sin \omega t\left|\mathbf{e}_{Y}\right\rangle \\
\left|\mathbf{e}_{2}(t)\right\rangle=-\sin \omega t\left|\mathbf{e}_{X}\right\rangle+\cos \omega t\left|\mathbf{e}_{Y}\right\rangle
\end{array}\right.
$$

La matrice associée au polariseur fixe est

$$
M_{X}=\left|\mathbf{e}_{X}><\mathbf{e}_{X}\right|,
$$

de sorte que le photomultiplicateur détecte une intensité lumineuse :

$$
\begin{aligned}
I_{\mathrm{d}} & =\operatorname{Tr}\left\{M_{X} M_{\varphi}(t) \pi M_{\varphi}^{+}(t) M_{X}^{+}\right\} \\
& =\left\langle\mathbf{e}_{a}(t)|\pi| \mathbf{e}_{a}(t)\right\rangle
\end{aligned}
$$

où $\left|\mathbf{e}_{a}(t)>=M_{\varphi}^{+}(t)\right| \mathbf{e}_{X}>$ est la polarisation correspondant à l'analyseur à l'instant $t ; \mid \mathbf{e}_{a}(t)>$ peut encore s'écrire :

$$
\begin{aligned}
\left|\mathbf{e}_{a}(t)\right\rangle=\mathrm{e}^{-i \varphi / 2} & {\left[\cos \frac{\varphi}{2}+i \sin \frac{\varphi}{2} \cos 2 \omega t\right]\left|\mathbf{e}_{X}\right\rangle+} \\
& +i \mathrm{e}^{-i \varphi / 2} \sin \frac{\varphi}{2} \sin 2 \omega t\left|\mathbf{e}_{Y}\right\rangle
\end{aligned}
$$

En reportant (2), (3), (7) dans (6), on obtient :

$$
I_{\mathrm{d}}=I_{\lambda^{\prime}}+\left(I_{\lambda}-I_{\lambda^{\prime}}\right)\left|<\mathbf{e}_{a}(t)\right| \mathbf{e}_{\lambda}>\left.\right|^{2}
$$

avec :

$$
\begin{aligned}
&\left|<\mathbf{e}_{a}(t)\right| \mathbf{e}_{\lambda}>\left.\right|^{2}= \\
&= \frac{1}{2}\left\{\sin ^{2} \frac{\varphi}{2}+2 \cos ^{2} \frac{\varphi}{2}\left|<\mathbf{e}_{X}\right| \mathbf{e}_{\lambda}>\left.\right|^{2}\right\}+ \\
&+ \frac{1}{2} \sin \varphi \sin 2 \omega t\left\{\left|<\mathbf{e}_{+}\right| \mathbf{e}_{\lambda}>\left.\right|^{2}-\right. \\
&+ \frac{1}{2} \sin ^{2} \frac{\varphi}{2}\left\{\operatorname { c o s } 4 \omega t \left[\left|<\mathbf{e}_{X}\right| \mathbf{e}_{\lambda}>\left.\right|^{2}-\right.\right. \\
&+\left.-\left|<\mathbf{e}_{Y}\right| \mathbf{e}_{\lambda}>\left.\right|^{2}\right] \\
&= \frac{1}{2}\left[1+\left.\cos 2 \psi\right|^{2}\right\} \\
&+ \frac{1}{2} \sin \varphi \sin 2 \psi \sin 2 \theta \sin 2 \omega t \\
&+ \sin ^{2} \frac{\varphi}{2}\left[\frac{1}{2} \cos \left(4 \omega t-2 \psi \mathbf{e}_{u}\left|\mathbf{e}_{\lambda}>\right|^{2}-\mid<\mathbf{e}_{\lambda}\right]\right\} \\
&\left.-\sin ^{2} \theta \sin 2 \psi \sin 4 \omega t\right] .
\end{aligned}
$$

Deux modulations apparaissent dans le signal délivré par le photomultiplicateur; leurs pulsations sont $2 \omega$ et $4 \omega$.

a) Modulation de pulsation $2 \omega$. - C'est cette modulation que nous avons utilisée dans nos expériences; elle possède quelques propriétés intéressantes :

— sa phase ne dépend ni de $\varphi$, ni de $\psi$, ni de $\theta$. Cette propriété est très commode pour effectuer le réglage de phase d'un système de mesure utilisant un détecteur synchrone ; ce réglage, fait pour une polarisation $\left|\mathbf{e}_{\lambda}\right\rangle$ quelconque et une longueur d'onde quelconque, reste toujours valable, même si la lame tournante n'est pas exactement quart d'onde,

- son amplitude est proportionnelle à $\sin \varphi$; au voisinage de $\varphi=\pi / 2$ (quart d'onde), cette amplitude est stationnaire et le choix de la lame biréfringente n'est pas critique,

- l'amplitude de cette modulation est aussi proportionnelle à $\sin 2 \psi \sin 2 \theta$. Cette quantité est extrémale lorsque $\left|\mathbf{e}_{\lambda}\right\rangle$ correspond à une des deux polarisations circulaires, droite ou gauche ; elle est nulle lorsque $\left|\mathbf{e}_{\lambda}\right\rangle$ correspond à une polarisation linéaire. La quantité $\sin 2 \psi \sin 2 \theta$ s'interprète comme le " degré de polarisation circulaire » de $\left|\mathbf{e}_{\lambda}\right\rangle$, ou encore comme la valeur moyenne de la projection sur $O Z$ du moment cinétique de spin des photons de polarisation $\mid \mathbf{e}_{\lambda}>$ (à un facteur $\hbar$ près).

- si $\varphi=\pi / 2, \theta=\psi=\pi / 4$ (cas de certaines de noexpériences).

$$
\begin{aligned}
I & =I_{\lambda^{\prime}}+\left(I_{\lambda}-I_{\lambda^{\prime}}\right) \cos ^{2}\left(\omega t-\frac{\pi}{4}\right) \\
& =\frac{1}{2}\left(I_{\lambda}+I_{\lambda^{\prime}}\right)+\frac{1}{2}\left(I_{\lambda}-I_{\lambda^{\prime}}\right) \sin 2 \omega t .
\end{aligned}
$$


Le taux de polarisation circulaire est égal au taux de modulation du signal délivré par le photomultiplicateur.

b) Modulation de pulsation $4 \omega$. - Cette modula- tion comprend deux composantes en quadrature de phase l'une par rapport à l'autre ; ces deux composantes sont proportionnelles au "degré de polarisation linéaire » de la lumière incidente soit suivant $O X$ et $O Y$, soit suivant les bissectrices de ces axes.

\section{Bibliographie}

[1] Kastler (A.), J. Physique Rad., 1950, 11, 255 ; J. Opt. Soc. Am., 1957, 47, 460.

Voir, par exemple, les références citées dans :

Brossel (J.), Quantum Optics and Electronics, Cours sur le pompage optique à l'école des Houches, 1964 (Gordon and Breach).

Cohen-Tannoudi (C.) et Kastler (A.), Progress in Optics, Vol. V (North Holland Publishing Co., Amsterdam).

[2] Cagnac (B.), Thèse, Paris, 1962, Ann. de Phys., 1961, 6, 467 ; J. Physique Rad., 1958, 19, 863.

[3] Lehmann (J. C.) et Brossel (J.), C. R. Acad.Sci. (Fr.), 1964, 258, 869.

LehmanN (J. C.) et Cohen-Tannoudit (C.), $C . R$. Acad. Sci. (Fr.), 1964, 258, 4463.

[4] Spence (D. W.) et Mc Dermott (M. N.), Phys. Lett., 1967, 24A, 430.

[5] Lehmann (J. C.), J. Physique, 1964, 25, 809 ; Thèse, Paris, 1966 ; Ann. de Phys., 1967, 2, 345 ; Phys. Rev. 1969, 178, 153.

[6] Barrat (J. P.) et Cohen-Tannoudji (C.), J. Physique Rad., 1961, 22, 329 et 443.

[7] Cohen-Tannoudji (C.), Thèse, Paris, 1962 ; Ann. de Phys., 1961, 7, 423 et 469.

[8] Faroux (J. P.) et Brossel (J.), C. R. Acad.Sci. (Fr.), $1965,261,3092 ; 1966,262 B, 41$ et $1385 ; 1966$, 263B, 612;1967, 264B, 1452 et $1573 ; 1967$, 265B, 393.

Faroux (J. P.), Thèse, Paris, 1969.

[9] Omont (A.), J. Physique, 1965, 26, 26 ; Thèse, Paris, 1967.

[10] Grossetête (F.), J. Physique, 1964, 25, 383 ; Thèse, Paris, 1967.

[11] Pebay-Peyroula (J. C.), Thèse, Paris, 1969 ; J. Physique Rad., 1959, 20, 669 et 721.

[12] Descoubes (J. P.), Thèse, Paris, 1967 ; C. R. Acad. Sci. (Fr.), 1964, 259, 327 ; 1964, 259, 3733 ; 1965, 261, 916.

[13] Colegrove (F. D.), Schearer (L. D.) et Walters (G. K.), Phys. Rev., 1963, 132, 2561

[14] Hanle (W.), Zeit. Phys., 1924, 30, 93.

voir aussi : Mrtchell (A. C. G.) et Zemansky (M. W.) ( Resonance Radiation and excited atoms), Cambridge University Press, 1934 et 1961.

[15] Grossetête (F.), Laloê (F.), Cohen-Tannoudji (C.) et Brossel (J.), C. R. Acad. Sci. (Fr.), 1967, 265B, 1247.

[16] Laló̂ (F.), Thèse $3^{e}$ cycle, Paris 1968.

[17] Dyakonov (M. I.) et Perel' (V. I.), Soviet Physics JETP, 1965, 20, 997.

[18] Omont (А.), J. Physique, 1965, 26, 576 ; voir aussi la référence [9].

[19] Nedelec (O.), Thèse, Grenoble, 1966.

[20] Decomps (B.) et Dumont (M.) I. E. E. E. J. Quant. Elect., 1968, 4, 916. [21] Capeller (V.) et Dellit (L.), Phys. Lett., 1968, 26A,
[22] Cohen-Tannoudi (C.) et Lalố (F.), J. Physique, $1967,28,505$ et 722 .

[23] Laloê (F.), Leduc (M.) et Minguzzi (P.), J. Physique, 1969, 30, 277 et 341.

[24] Williams (W. L.) et Fry (E. S.), Phys. Rev. Lett., 1968, 20, 1335.

[25] Greenhow (R. C.), Phys. Rev., 1964, 136, 660.

[26] Schearer (L. D.), Thèse, Rice University, 1966.

[27] Dehmelt (H. G.), Phys. Rev. 1957, 105, 1487.

[28] Fred (M.), Tomkins (F. S.), Brody (J. K.) et HamerMESH (M.), Phys. Rev., 1951, 82, 406.

[29] Lalố (F.), C. R. Acad. Sci. (Fr.), 1968, 267, 208. Pavlovic (M.) et Laló̈ (F.), C. R. Acad. Sci. (Fr.), 1969,1436 et 1485.

[30] Maujean (M.) et Descoubes (J. P.), C. R. Acad. Sci. (Fr.), 1967, 264, 1653.

[31] Bates (D. R.) et Darmgand (A.), Phil. Trans. A., 1950, 242, 101.

[32] Bennett (W. R.), Kindlmann (J. P.) et Mercer (G. N.), Applied Optics 1965, Suppl. 2. (C Chemical Lasers ), 34.

[33] Osherovich (A. L.) et Verolainen (Ya. F.), Opt. and Spect., 1968, 24, 81.

[34] Cette durée de vie $\tau \simeq 1,8 \times 10^{-9} \mathrm{~s}$ a été calculée à partir des forces d'oscillateur données par :

Schiff (B.) et Pekeris (C. L.), Phys. Rev., 1964, 134A, 638.

Un calcul théorique de l'ordre de grandeur de cette durée de vie, donnant $\tau \simeq 2 \times 10^{-9} \mathrm{~s}$ a aussi été effectué par :

Heron (S.), Mc Whirter (R. W. P.) et Rhoderick (E. H.), Proc. Roy. Soc. A, 1966, 234, 565.

[35] Cette structure hyperfine peut être évaluée à partir des calculs théoriques de Descourbes [12], et de la connaissance de l'écart énergétique entre les niveaux $3{ }^{1} P$ et $3{ }^{3} P$ [36].

[36] Moore (L. E.), Atomic Energy Levels, Vol. I (NBS 467, 1949).

[37] Gamblin (R. L.) et Carver (T. R.), Phys. Rev., 1965, 138, 946.

[38] Schearer (L. D.) et Walters (G. K.), Phys. Rev., 1965, 139, 1398.

[39] Decomps (B.), Pebay-Reyroula (J. C.) et Brossel (J.), C. R. Acad. Sci. (Fr.), 1961, 252, 537.

Descoubes (J. P.), Decomps (B.) et Brossel (J.), C. $R$. Acad. Sci. (Fr.), 1964, 258, 4005.

[40] Decomps (B.), Thèse $3^{\mathrm{e}}$ cycle, Paris 1960.

[41] Bessis (N.), Lefebvre-Brion (H.) et Moser (C. M.), Phys. Rev., 1964, 135, 957.

[42] Bridgett (K. A.) et King (T. A.), Proc. Phys. Soc., 1967, 92, 75.

[43] Giroud (M.), Lombardi (M.) et Pebay-Peyroula (J. C.), J. Physique, 1969, 30, 789.

[44] DrTIL (W.), Zeit. Naturforch., 1969, 24A, 350.

[45] Buchhaupt (K.), Zeit. Naturforch., 1969, 24A, 1058. 